\title{
Processus de mise en place et d'évolution de la couverture sédimentaire superficielle de la plate-forme nord-aquitaine
}

\author{
Pierre CIRAC $^{\text {a* }}$, Serge BERNE ${ }^{\text {b }}$, Patrice CASTAING ${ }^{\text {a }}$, Olivier WEBER ${ }^{a}$ \\ ${ }^{a}$ Département de géologie et océanographie, UMR Epoc 5805-CNRS, université Bordeaux-I, \\ avenue des facultés, 33405 Talence cedex, France \\ b Institut français de recherche pour l'exploitation de la mer, Ifremer, Centre de Brest, BP 70, \\ 29280 Plouzané cedex, France
}

Reçu le 26 novembre 1998, reçu en forme révisée le 10 juin 1999, accepté le 7 juillet 1999

\begin{abstract}
Sedimentary processes and facies on the northern Aquitaine shelf, France. The Aquitaine shelf in France is a storm-dominated shelf similar to the Californian shelf in the US. It exhibits a thin veneer (about 1-2 m) of sandy sediments overlying coarse-grained deposits. This surficial sand sheet is shaped into various bed forms corresponding to the contrasted physical processes operating at different water depths and time-scales. These bed forms are: a) sand patches separated by depressions or 'furrows', b) large transverse dunes and c) large wave ripples. The overall architecture of the sand cover is considered as resulting from the stacking of several storm beds. During the last post-glacial sea-level rise, the magnitude of reworking processes progressively decreased as a result of increasing water depth. This is demonstrated by the overall upward thinning and fining of elementary storm beds. Since ca. 4000 years BP, sea-level has remained approximately constant and reworking mainly affected the upper 1-2 m of sediment on the inner shelf. Deeper than about 20-30 m below sea-level, present-day reworking appears as being limited to the upper tenths of centimeters, during major storms. Winnowing of Pleistocene coarse-grained sediments as well as of transgressive sand patches provide the material for this thin upperlying highstand deposit, still in equilibrium with present processes. (C) 2000 Ifremer / CNRS / IRD / Éditions scientifiques et médicales Elsevier SAS
\end{abstract}

\section{sedimentology / hydrodynamic processes / bedforms / sequence deposits / northern Aquitaine shelf}

Résumé - À l'image de la plate-forme californienne des États-Unis, le plateau continental nord-aquitain est dominé par les tempêtes. Les mesures in situ y sont rares mais les observations et les prélèvements sur le fond sont nombreux. Ce plateau est comparé aux plates-formes américaines étudiées sur le plan des processus physiques et des corps sédimentaires qu'ils engendrent. La couverture superficielle nord-aquitaine, épaisse d'1 à $2 \mathrm{~m}$, présente en surface des formes sédimentaires de trois échelles de grandeur liées à des processus hydrodynamiques différents. Par ordre de taille décroissante : a) des placages sableux et des couloirs, b) des grandes mégarides et c) des mégarides d'oscillation engendrées par la houle. L'organisation interne de la couverture superficielle, constituée de séquences élémentaires superposées, est attribuée à des processus de tempête. Lors de la dernière remontée marine post-glaciaire, l'intensité des remaniements diminue. Elle se marque par une diminution de granulométrie et d'épaisseur des séquences élémentaires, tant de la côte vers le large que de la base vers le sommet des placages sableux. Au cours des derniers 4000 ans BP (Holocène terminal), le niveau marin reste constant. L'épaisseur de remaniement est importante ( 1 à $2 \mathrm{~m})$ sur le proche plateau interne. Au-delà de 20 à $30 \mathrm{~m}$ de profondeur, le remaniement n'affecte que les premiers décimètres

\footnotetext{
* Correspondence and reprints:
} 
de la couverture sédimentaire. Cette dynamique hydrosédimentaire conduit, par remaniement des placages sableux et par vannage des niveaux grossiers de la base, à la constitution d'une formation résiduelle dite de « haut niveau ». (C) 2000 Ifremer/CNRS/IRD/Éditions scientifiques et médicales Elsevier SAS

sédimentologie / processus hydrodynamiques / formes sédimentaires / séquences de dépôt / plate-forme nordaquitaine

\section{INTRODUCTION}

Le plateau continental nord-aquitain (figure 1) qui s'étend de l'estuaire de la Gironde $\left(45^{\circ} 45^{\prime} \mathrm{N}\right)$ au bassin d'Arcachon $\left(44^{\circ} 25^{\prime} \mathrm{N}\right)$ est considéré comme une plate-forme en zone tempérée dominée par les tempêtes et les très fortes houles [3, 11, 49]. La couverture sédimentaire superficielle est formée en grande partie par des placages sableux peu épais (en moyenne de l'ordre de 1 à $2 \mathrm{~m}$ ) et plus ou moins discontinus sur le substrat meuble pléistocène. Elle serait en équilibre hydrodynamique avec les processus actuels d'érosion/dépôt [50]. Dans la séquence de dépôt quaternaire mise en évidence par Cirac et al. [14], cette couverture superficielle correspond à l'unité supérieure de remaniement de la période de haut niveau marin. L'étude de la répartition des formes sédimentaires, de leur morphologie et de leurs structures internes permet de saisir les processus qui contrôlent la dynamique sédimentaire sur la plate-forme.

Comme l'ont montré Turcq [49], Collotte [16] et Arbouille [4], les formes sédimentaires les plus fréquemment observées à la surface du plateau nordaquitain sont des dépressions ou des couloirs (ou sillons) encadrés par des placages sableux. Le fond de ces couloirs est constitué de sédiment grossier (sables grossiers, graviers et galets). Des formes semblables ont été décrites sur des plates-formes dominées par les tempêtes, notamment sur les plateaux sud-est africain [19], nord-ouest africain [35], américain nordatlantique [9, 28, 32, 45], canadien [21], californien $[10,25,26]$. De nombreuses observations et mesures physiques ont été effectuées in situ au cours des dix dernières années, lors de très fortes tempêtes ou de cyclones sur les plates-formes américaines de l'Atlantique, du Pacifique et du golfe du Mexique permettant de faire avancer la connaissance des processus physiques liés aux tempêtes [36, 42] ainsi que leur interprétation en terme de dynamique sédimentaire [41, 47].

La plate-forme aquitaine présente des analogies certaines avec tous ces plateaux, en particulier avec les plateaux ouest et est américains. En effet, les processus hydrodynamiques aquitains sont largement dominés par les fortes tempêtes d'ouest, l'impact des courants tidaux étant limité à certaines zones estuariennes et côtières. La forte intensité des processus hydrodynamiques conduit à la dispersion des apports terrigènes fins actuels dont les dépôts peu importants sont confinés aux abords de l'estuaire de la Gironde (figure 2).

L'originalité du plateau aquitain réside dans l'orientation des fronts dépressionnaires des plus fortes tempêtes qui se propagent perpendiculairement à la côte (secteur ouest à sud-ouest), alors que sur les platesformes américaines, ils progressent, en général, obliquement par rapport au littoral. En revanche, contrairement aux travaux américains, nous ne disposons pas encore de mesures physiques effectuées au cours des tempêtes. Nous tenterons donc d'établir un schéma des processus qui contrôlent la dynamique sédimentaire de la couverture superficielle du plateau nord-aquitain en nous appuyant sur les travaux américains et sur ceux effectués sur le plateau aquitain $[6,11]$.

\section{CADRE PHYSIOGRAPHIQUE}

Le plateau continental nord-aquitain peut être subdivisé (figure 1) en trois zones géomorphologiques [49] :

- un plateau interne jusqu'à environ $40 \mathrm{~m}$ de profondeur, de topographie uniforme;

- un plateau médian, entre $40 \mathrm{~m}$ et $80 \mathrm{~m}$ de profondeur, dont la topographie est marquée par des buttes et des ondulations de 1 à $6 \mathrm{~m}$ d'amplitude étalées sur plusieurs centaines de mètres de largeur;

- un plateau externe, entre $80 \mathrm{~m}$ et $140 \mathrm{~m}$ de profondeur, de topographie régulière.

La figure $1 b$ montre que les limites entre ces zones géomorphologiques sont des anomalies de pente formant des abrupts dirigés vers le large. 


\section{CADRE HYDRODYNAMIQUE}

L'hydrodynamique sur la plate-forme aquitaine est dominée par les processus liés aux tempêtes [4, 50]. Les plus fortes tempêtes décennales, de secteur ouest
[37], engendrent des houles de 9 à $15 \mathrm{~m}$ d'amplitude et de 11 à $15 \mathrm{~s}$ de période. Les vitesses orbitales peuvent alors dépasser $1 \mathrm{~m} \cdot \mathrm{s}^{-1}$ près du fond [11] et, selon les profondeurs, varier de $0,5 \mathrm{~m} \cdot \mathrm{s}^{-1}$ à 2,5 $\mathrm{m} \cdot \mathrm{s}^{-1}$ [3]. La houle ne joue pas un rôle prépondérant
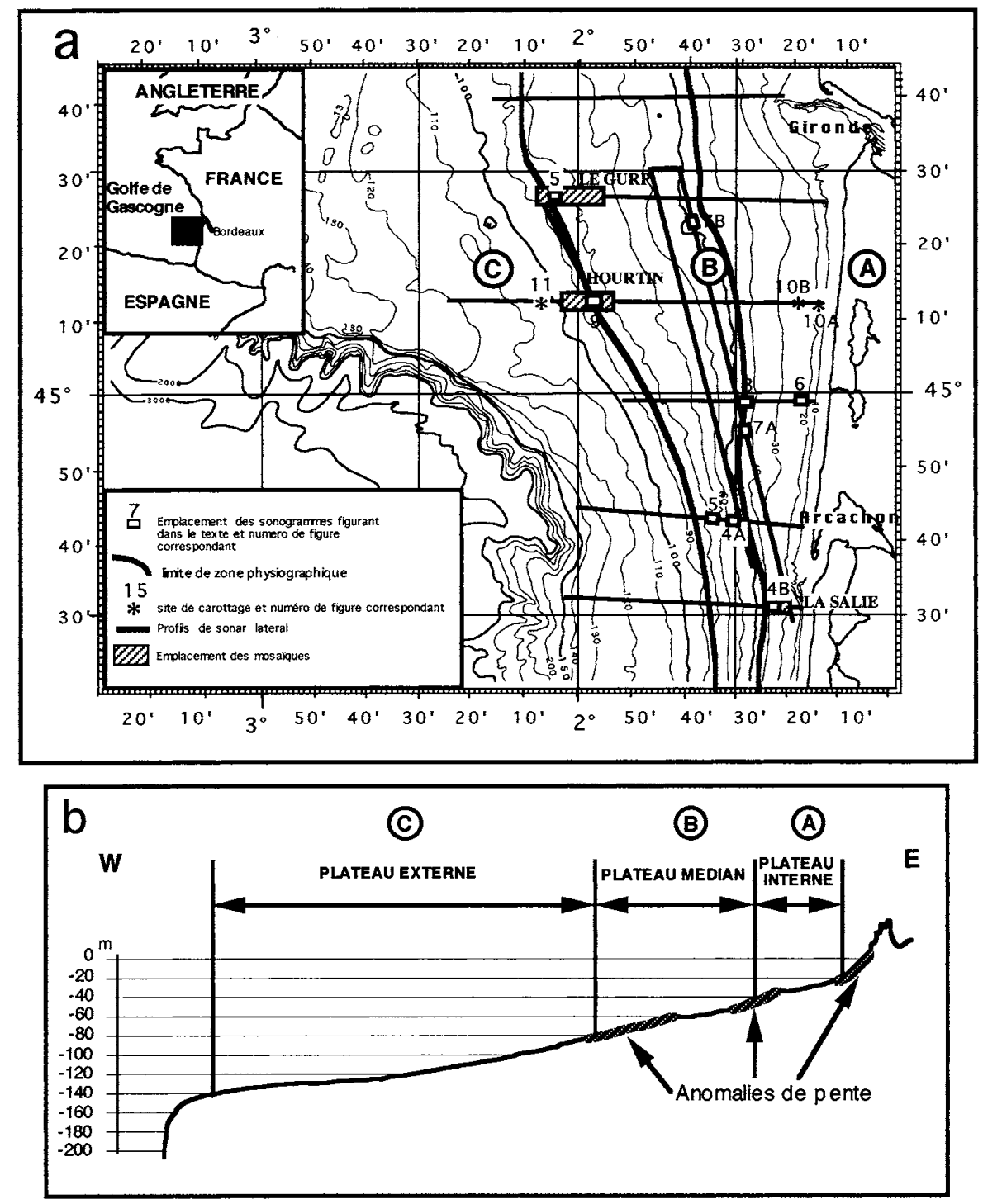

Figure 1. Physiographie du plateau continental nord-aquitain. a) Situation de la zone d'étude et bathymétrie de la plate-forme. A = plateau interne; $\mathrm{B}=$ plateau médian ; $\mathrm{C}=$ plateau externe. Emplacement des profils de sonar latéral et de sondeur de sédiment, des sites de carottages, de mosaïques de sonar latéral et des numéros de figures appelées dans le texte. b) Profil bathymétrique synthétique transverse à la plate-forme d'après Weber et al. [53].

Figure 1. Physiographic features of the northern Aquitaine continental shelf: a) Location of study area and bathymetric map. A = inner shelf; $\mathrm{B}=$ median shelf; $\mathrm{C}=$ outer shelf. Location of the side scan sonar and 3.5 seismic profiles, vibrocorers sites, sidescan sonar mosaics and figure numbers cited in the text. b) Schematic bathymetric profile across the shelf after Weber et al. [53]. 


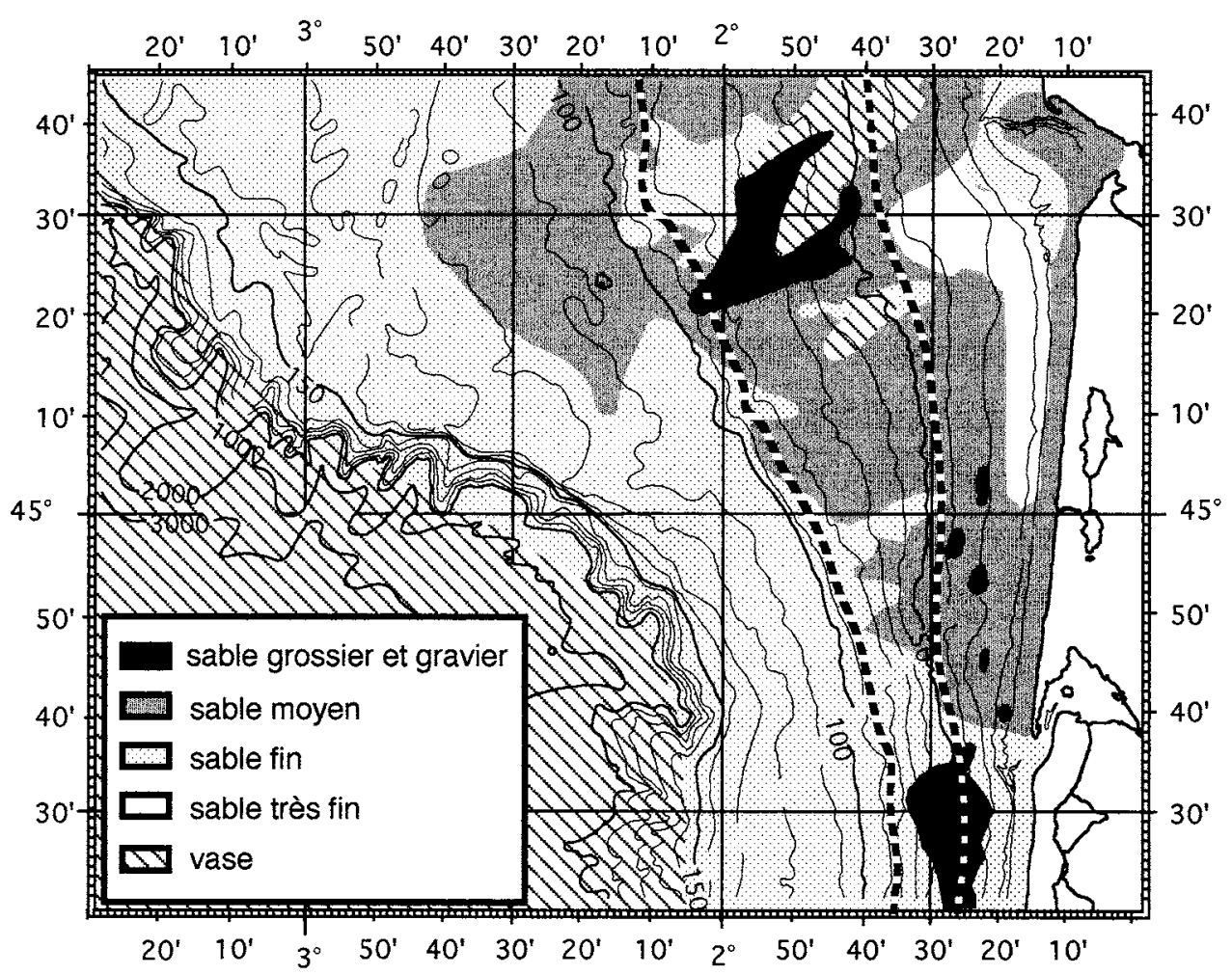

Figure 2. Répartition des sédiments sur le plateau continental nord-aquitain (modifié d'après Allen et Castaing [1]). Les tiretés épais correspondent aux limites de zone de la figure 1.

Figure 2. Surficial sediment distribution over the northern Aquitaine continental shelf (modified after Allen and Castaing [1]). Thick dashed lines correspond to the limits of shelf zonation defined in figure 1.

dans le transit des sédiments, sauf près des côtes, mais elle contribue à leur remise en suspension. Nous verrons, en revanche, que les courants combinés, induits par les tempêtes, ont un rôle primordial sur le fond.

Le tableau I synthétise les caractères hydrodynamiques des plateaux continentaux les plus étudiés. Parmi les plateaux dominés par les tempêtes, le plateau aquitain est de ceux dont l'énergie hydrodynamique est la plus élevée : les houles de longues périodes et de grandes amplitudes y induisent des vitesses orbitales sur le fond élevées. L'hydrodynamisme du plateau aquitain est tout à fait comparable à celui des plateaux américains atlantique et californien, ainsi qu'à celui du sud-est australien.

Les zones physiographiques définies plus haut peuvent également être caractérisées par des critères hydrodynamiques :

- la limite entre le plateau interne et la zone marginolittorale se situe vers $20 \mathrm{~m}$ de profondeur et correspond à la limite d'action des houles de beau temps [13] ;

- la limite entre plateaux interne et médian ne semble pas correspondre à une limite hydrodynamique particulière ;

- la limite entre plateaux médian et externe, située vers $80 \mathrm{~m}$, correspondrait à la limite d'action des très fortes houles de tempêtes décennales [11];

- sur le bord du plateau, les sédiments ne seraient que très exceptionnellement mis en oscillation par des houles de $15 \mathrm{~s}$ de période et d'environ $15 \mathrm{~m}$ d'amplitude [11]. 


\section{CADRE SÉDIMENTAIRE}

La couverture sédimentaire du plateau aquitain a été étudiée par bon nombre d'auteurs [1, 4, 7, 16, 29, 49]. La répartition des faciès sédimentaires (figure 2) montre qu'elle est constituée, en grande partie, de sables moyens et fins avec une évolution du nord au sud. Au nord, les sables moyens dominent; ils s'étendent sur les plateaux interne et médian et débordent largement sur le plateau externe. Au sud, l'extension des sables moyens diminue progressivement au bénéfice des sables fins qui couvrent l'ensemble du plateau au sud des passes du bassin d'Arcachon.

À proximité de l'embouchure de la Gironde, et en liaison avec les apports actuels ou sub-actuels de celle-ci, on observe des recouvrements de vases et de sables très fins [30]. Localement, à la faveur de dépressions, apparait un substrat sablo-graveleux [50].

La couverture sédimentaire du plateau du golfe de
Gascogne est constituée d'éléments [3] apportés par les fleuves sur la plate-forme lors des régressions glacio-eustatiques qui se sont succédées au cours du Quaternaire. Une étude sismique [14] a montré qu'elle correspond à une seule séquence de dépôt d'une vingtaine de mètres d'épaisseur. Cette couverture sédimentaire comprend trois unités :

1) une unité de base consistant en des remplissages d'incision fluviatile en période de bas niveau marin;

2) une unité " transgressive », représentant l'essentiel de l'épaisseur (20 m à $30 \mathrm{~m}$ ), constituée d'une superposition de nappes rétrogradantes;

3) une unité superficielle d'environ $2 \mathrm{~m}$ d'épaisseur.

L'unité transgressive, selon le modèle préconisé par Aloisi et al. [3], est constituée de matériaux reliques remaniés lors de leur redistribution sur le plateau en

Tableau I. Intensité maximum des phénomènes hydrodynamiques mesurée sur différents plateaux continentaux d'après les travaux de: (1) Aloisi et al. [3], Castaing [11], Arbouille [4]; (2) Auffret et al. [5] ; (3) Drapper [18] Channon et Hamilton [13] ; (4) Drapper [18];

(5) Swift et Freeland [45], Forbes et Boyd [21], Knebel et al. [28], Swift et al. [47]; (6) Roy et al. [40] ; (7) Hunter et al. [25],

Cacchione et al. [10]; (8) Snedden et al. [42].

Table I. Maximum intensity of hydrodynamic processes on different continental shelves after: (1) Aloisi et al. [3], Castaing [11], Arbouille [4]; (2) Auffret et al. [5]; (3) Drapper [18], Channon and Hamilton [13]; (4) Drapper [18]; (5) Swift and Freeland [45], Forbes and Boyd [21], Knebel et al. [28], Swift et al. [47]; (6) Roy et al. [40]; (7) Hunter et al. [25], Cacchione et al. [10]; (8) Snedden et al. [42].

\begin{tabular}{|c|c|c|c|c|c|c|c|}
\hline \multirow{2}{*}{$\begin{array}{l}\text { Plateaux } \\
\text { continentaux }\end{array}$} & \multirow{2}{*}{$\begin{array}{l}\text { Environnement } \\
\text { tidal }\end{array}$} & \multicolumn{3}{|c|}{ Houle } & \multicolumn{3}{|c|}{ Courant } \\
\hline & & $\begin{array}{l}\text { Période } \\
\text { (s) }\end{array}$ & $\begin{array}{l}\text { Amplitude } \\
(\mathrm{m})\end{array}$ & $\begin{array}{l}\text { Vitesse orbitale } \\
\left(\mathrm{m} \cdot \mathrm{s}^{-1}\right)\end{array}$ & Processus & $\begin{array}{l}\text { Vitesse (surf.) } \\
\left(\mathrm{m} \cdot \mathrm{s}^{-1}\right)\end{array}$ & $\begin{array}{l}\text { Vitesse (fond) } \\
\left(\mathrm{m} \cdot \mathrm{s}^{-1}\right)\end{array}$ \\
\hline $\begin{array}{l}\text { Golfe de Gascogne } \\
\text { (1) }\end{array}$ & $\begin{array}{l}\text { Meso à } \\
\text { macrotidal }\end{array}$ & $8-15$ & $9-15$ & $>0,8^{\mathrm{b}}$ & tempêtes & $0,2-0,55$ & $0,1-0,30^{\mathrm{c}}$ \\
\hline Manche (2) & $\begin{array}{l}\text { macro à } \\
\text { mégatidal }\end{array}$ & & 7.6 & & $\begin{array}{l}\text { marée \& } \\
\text { tempêtes }\end{array}$ & $0,4-3$ & $0,4-0.6^{\mathrm{d}}$ \\
\hline Mer celtique (3) & $\begin{array}{l}\text { macro à } \\
\text { mégatidal }\end{array}$ & & & $>0,45^{\mathrm{b}}$ & $\begin{array}{l}\text { marée \& } \\
\text { tempêtes }\end{array}$ & $1-3$ & $0,2-0,5^{\mathrm{c}}$ \\
\hline Mer du Nord (4) & macrotidal & $6-8$ & $5-8$ & $0,26-0,48$ & $\begin{array}{l}\text { marée \& } \\
\text { tempêtes }\end{array}$ & $0,5-1,5$ & $0,3-0,9^{\mathrm{c}}$ \\
\hline $\begin{array}{l}\text { Est des Etats-Unis } \\
\text { (5) }\end{array}$ & $\begin{array}{l}\text { micro à } \\
\text { mesotidal }\end{array}$ & $6-13$ & 8 & $0,2-1$ & tempêtes & 1 & $0,05-0,6^{\mathrm{c}}$ \\
\hline Sud-est Australie (6) & $\begin{array}{l}\text { micro à } \\
\text { mesotidal }\end{array}$ & & $4^{\mathrm{a}}$ & & tempêtes & & \\
\hline Californie (7) & microtidal & 25 & 9 & 1,9 & tempêtes & & $0,18-0,35$ \\
\hline Texas (8) & microtidal & $6-12$ & $2-8$ & & ouragans & 0,5 & $0,5-2$ \\
\hline
\end{tabular}

${ }^{\text {a }}$ Pendant $1 \%$ du temps maximum $16 \mathrm{~m}$.

${ }^{\mathrm{b}}$ Pendant $10 \%$ du temps.

c À $1 \mathrm{~m} \mathrm{du}$ fond.

d À $0.7 \mathrm{~m} \mathrm{du}$ fond. 
phase transgressive. L'unité superficielle correspond aux remaniements par les agents hydrodynamiques contemporains (Holocène terminal) dont les tempêtes assument le rôle primordial, ainsi que nous allons le voir.

Cette couverture superficielle, objet du présent travail, est peu accessible à l'étude sismique en raison de sa faible épaisseur. Nous utiliserons des méthodes d'imagerie acoustique pour l'étude des formes sédimentaires sur la surface du fond (bed forms) et des carottages pour l'étude des structures sédimentaires internes.

\section{MÉTHODES UTILISÉES}

Les données utilisées proviennent des nombreuses missions effectuées depuis 1982, dans le cadre des programmes Fasec et Geodep, par le département de géologie et océanographie de l'université Bordeaux-I (UMR Epoc 5805) en collaboration avec le laboratoire des environnements sédimentaires de l'Ifremer.

L'étude morphologique des corps sédimentaires a été effectuée à l'aide d'un sondeur bathymétrique Simrad EK400, d'un sondeur de sédiment EDO Western 3,5 kHz puis 2,5 kHz ainsi que d'un sonar latéral EGG 272 associé à un enregistreur SMS 960. Ces mesures ont été étalonnées par de nombreuses bennes et carottes courtes. Le positionnement de la route du navire et des points de prélèvement a été assuré par un système de radiolocalisation, Toran puis Syledis.

Les faciès et les structures sédimentaires ont été étudiés au moyen de vibro-carottages d'une longueur maximale de $2,8 \mathrm{~m}$. Les carottes prélevées ont fait l'objet, après ouverture au laboratoire, d'une description détaillée, de photographies, de clichés aux rayons $\mathrm{X}$, d'analyses granulométriques et de 29 datations au radiocarbone sur des valves de lamellibranches.

Les données de sonar latéral et de sismique ont été acquises le long de profils transversaux et longitudinaux représentés sur la figure 1, ainsi que l'emplacement de trois zones ayant donné lieu à des mosaïques de sonar latéral et la position des vibrocarottages.

\section{RÉSULTATS}

\subsection{Formes sédimentaires}

Les formes sédimentaires observées sur le plateau nord-aquitain peuvent être classées selon trois échelles de grandeur:

- les formes de grande taille (longueur L de l'ordre de plusieurs, voire de la dizaine de kilomètres; largeur 1 de l'ordre de plusieurs centaines de mètres à quelques kilomètres) correspondant à des placages sableux peu épais ( $2 \mathrm{~m}$ maximum) et entrecoupés de zones en dépression (couloirs, sillons, cuvettes) dont le fond est constitué de sédiment grossier;

- les formes de taille moyenne, représentées par de grandes mégarides selon la définition de Swift et al. [46] ou de petites dunes au sens de Berné et al. [8] (longueur L de $100 \mathrm{~m}$ à $1000 \mathrm{~m}$; largeur 1 de 10 à $100 \mathrm{~m}$; amplitude de 1 à $3 \mathrm{~m})$;

- les formes de petite taille (de 1 à $8 \mathrm{~m}$ de longueur d'onde et de $0,10 \mathrm{~m}$ à $0,4 \mathrm{~m}$ d'amplitude), représentées par des mégarides d'oscillation.

\subsubsection{Formes sédimentaires de grande taille}

Parmi ces formes sédimentaires, on distingue deux aspects :

- Les bandes de graviers et sables grossiers correspondant à un substrat grossier qui tapisse le fond de dépressions.

Les bandes sont plus abondantes et mieux développées sur les zones d'anomalie de pente (figure 1) correspondant à une accentuation de la pente topographique. Les dépressions qui n'excèdent pas $2 \mathrm{~m}$ de profondeur peuvent avoir des formes quelconques, mais le plus souvent allongées sensiblement nord-sud, parallèlement à la ligne de rivage (figure 3). Les placages sableux qui les encadrent prennent parfois l'aspect de rubans sableux. Ce type de placages sableux peut être comparé aux placages sableux transverses (transverse sand patches) décrits par Kenyon [27].

Le bord des dépressions peut être net et abrupt ou plus fréquemment, l'un des bords a un versant plus doux, parfois festonné (figure 4a). Il arrive que la limite entre les deux faciès acoustiques, sombre et 
clair, soit diffuse, peu nette, constituée d'un voile sableux et fréquemment accompagnée de mégarides de houle parallèles à cette limite (figure 4b). Les couloirs et placages prennent alors un aspect dissymétrique. Souvent, le bord abrupt est dirigé vers le large, comme sur la figure $4 b$, mais cette disposition n'est pas une constante sur le plateau aquitain.

Les profils de sondeur de sédiment font apparaître, à la base des placages sableux (figure 4), un réflecteur en continuité avec le substrat grossier des dépressions.

- Les linéations (figure 5) se rencontrent principalement dans les sables fins du plateau externe, entre 80 et $140 \mathrm{~m}$ de fond. Ces formes sédimentaires, de type sillons érosifs (erosional furrows) ou sillons longitudinaux (longitudinal furrows), présentent, sur le plateau nord-aquitain, des longueurs de 200 à $300 \mathrm{~m}$, un espacement de 20 à $50 \mathrm{~m}$ et une orientation N100 à N120. Elles sont rectilignes et parallèles avec des profondeurs de 1 à $2 \mathrm{~m}$. Le fond des linéations est, ici aussi, occupé par des sédiments grossiers.

\subsubsection{Formes sédimentaires de taille moyenne}

Ces formes sont représentées par des corps sédimentaires présents, la plupart du temps, sur le plateau interne jusqu'à $40 \mathrm{~m}$ de profondeur. Elles consistent (figure 6) en ondulations asymétriques de 10 à $100 \mathrm{~m}$

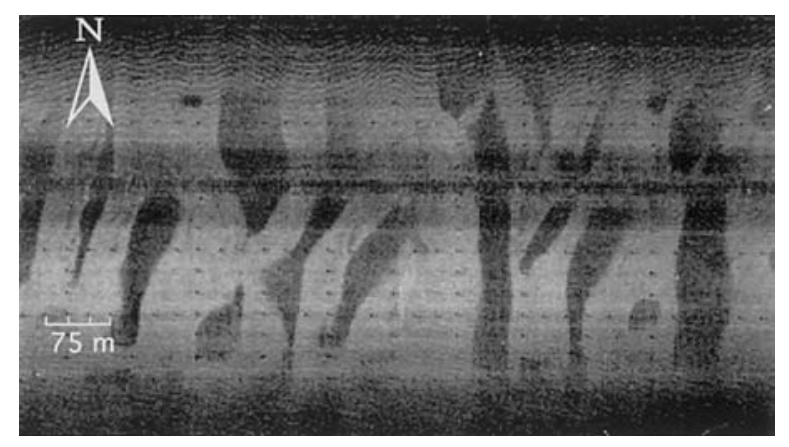

Figure 3. Photographie d'un enregistrement de sonar latéral sur le plateau continental nord-aquitain montrant un placage sableux (écho-faciès clair) parcouru par des dépressions allongées parallèlement, selon une direction nord-sud et dont le fond est tapissé d'un sédiment grossier (écho-faciès sombre).

Figure 3. Sonogram of a sand patch (light echofacies) marked by north-south elongated depressions covered by coarse sediment (dark echofacies). d'espacement dont les crêtes, longues et sinueuses, parfois arquées, sont orientées nord-sud. Leur hauteur $\mathrm{H}$, est difficile à déterminer exactement sur les profils sismiques ou bathymétriques car inférieure à la définition des systèmes. On peut estimer, en se référant à Flemming [20], qu'elle est de l'ordre de 1 à $3 \mathrm{~m}$. Ces corps sédimentaires peuvent être considérés comme de petites dunes au sens de Berné et al. [8]. Elles caractérisent des zones de charriage où les conditions hydrodynamiques et un stock sableux suffisant permettent leur édification. La figure 6 montre que la face abrupte de ces dunes est dirigée vers l'est ; elles se déplaceraient donc vers le littoral.

\subsubsection{Formes sédimentaires de petite taille}

Ces formes sédimentaires consistent essentiellement en mégarides de houle et en structures de type « bombé».

Les mégarides de houle (figure 7a) sont des formes symétriques ou très légèrement dissymétriques ayant une crête rectiligne, parfois bifurquée. Elles ont été souvent décrites, sous le terme de wave megaripples $[17,46]$.

Leur longueur d'onde est comprise entre 1 et $3 \mathrm{~m}$, exceptionnellement, elle peut atteindre $8 \mathrm{~m}$. D'après les travaux de Allen [2], on estime leur amplitude entre 0,1 et $0,4 \mathrm{~m}$.

Les mégarides de houle sont des formes sédimentaires transverses engendrées par des courants oscillatoires près $\mathrm{du}$ fond. La vitesse critique d'entraînement nécessaire à la mise en mouvement d'un sédiment sableux grossier est de l'ordre de $60 \mathrm{~cm} \cdot \mathrm{s}^{-1}$, ce qui, pour une profondeur de $60 \mathrm{~m}$, correspond à des vagues de période de $15 \mathrm{~s}$ et 4 à $5 \mathrm{~m}$ d'amplitude [11]. Ce sont là des conditions de tempête.

Les mégarides se rencontrent sur l'ensemble du plateau nord-aquitain, quelle que soit la profondeur. On les observe plus particulièrement sur les fonds sablo-graveleux qui constituent le substrat des couloirs d'érosion. Leurs crêtes sont toujours orientées nord-sud et devraient donc être engendrées par les fortes tempêtes d'ouest ou de nord-ouest.

Des formes bombées ou en mamelons dans le sens de Guillocheau [23] ont été observées (figure 7b), de façon exceptionnelle, sur le plateau interne aquitain, entre 20 et $40 \mathrm{~m}$ [19]. Elles sont liées génétiquement aux stratifications bombées (hummocky cross stratifi- 


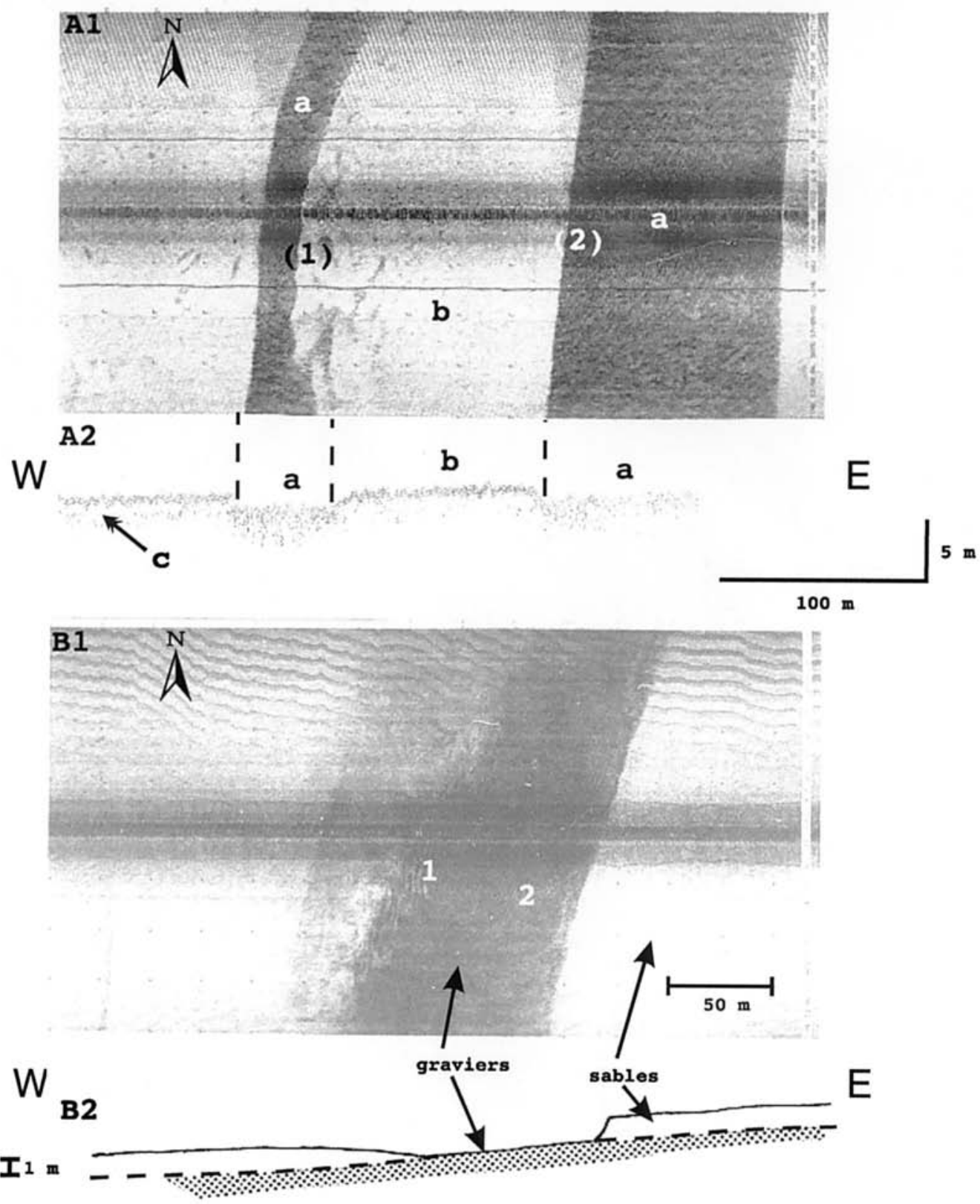

Figure 4. Sonogrammes (A1, B1) et profils de sondeur de sédiment (A2, B2) de placages sableux (position des images sur la figure 1 ; les franges parallèles en bordure de l'image correspondent à un bruit d'hélice). A : bandes de faciès acoustique sombre, constituées de sables grossiers et graviers (a) encadrées par les placages sableux (b) présentant à la base une réflexion (c) en continuité avec le fond des dépressions. Noter les limites parfois nettes et abruptes (2) et parfois festonnées et plus floues (1). B : dépression dissymétrique à substrat de gravier dont l'un des bords (est) est abrupt alors que l'autre (ouest) comporte une limite diffuse réduite à un voile sableux sur lequel on distingue un champ de mégarides de houle (1) : $\mathrm{L}=3$ à $4 \mathrm{~m}$. On note sur le reste de la dépression des mégarides plus petites (2): $\mathrm{L}=1 \mathrm{~m}$.

Figure 4. Sonograms (A1, B1) and $3.5 \mathrm{kHz}$ seismic profiles (A2, B2) of sand patches (see location in figure 1; (an artefact with parallel fringes visible at the side of the image is due to screw noise). A: stretches of dark echofacies made of coarse sand and gravel (a) surrounded by sand patches (b) showing a bottom reflector extending laterally to the lower part of the depressions. Note the sharp and steep limits sometimes festooned and fuzzy (1). B: dissymetrical depression of gravels with an abrupt edge toward the east and a gentle slope to the west of a thin sandy sheet covered with wavy megaripples (1): $\mathrm{L}=3$ to $4 \mathrm{~m}$. Other smaller megaripples are noted on the rest of the depression (2): $\mathrm{L}=1$ $\mathrm{m}$. 


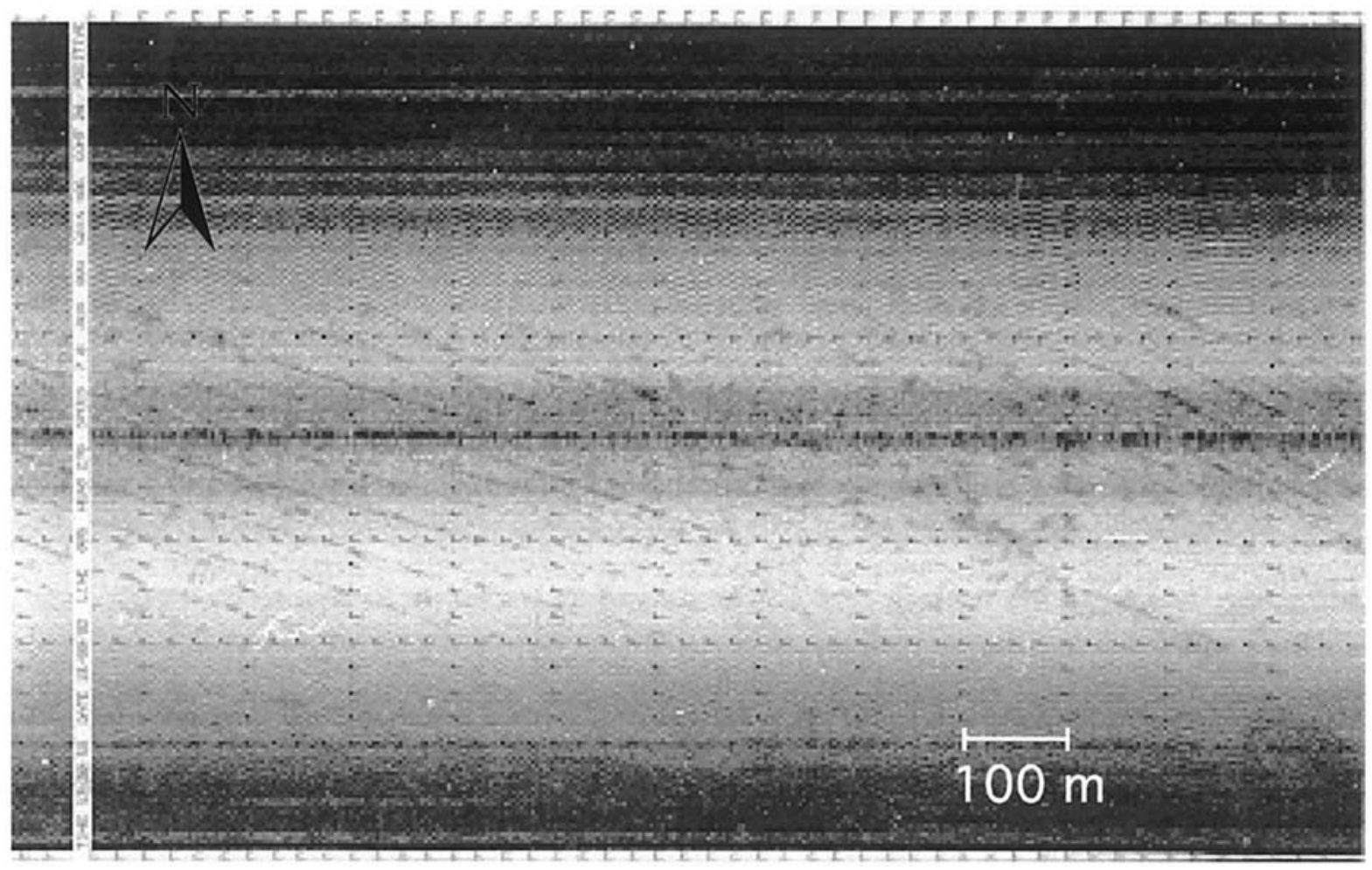

Figure 5. Champ de linéations parallèles et discontinues sur le plateau nord-aquitain externe.

Figure 5. Discontinuous and parallel lineations on the northern Aquitaine outer shelf.

cation) décrites par Harms et al. [24] et Swift et al. [44] et étudiées en détail plus loin. Elles ont, en moyenne, une longueur d'onde de $10 \mathrm{~cm}$ et une amplitude de 1 à $2 \mathrm{~cm}$, et seraient induites par la houle [16].

\subsection{Faciès sédimentaires}

Les profils de sondeur de sédiment $(2,5$ et $3,5 \mathrm{kHz}$, figure 8) permettent d'individualiser une réflexion qui marque une surface d'érosion supérieure en pente irrégulière vers le large, sous-jacente à une unité sédimentaire supérieure (unité A de Cirac et al. [14]). Cette formation superficielle, relativement peu épaisse (en moyenne $2 \mathrm{~m}$ ), constitue soit des placages sableux continus et relativement homogènes, soit des « formes sédimentaires » décrites précédemment dans l'analyse morphologique.
L'étude de 48 vibro-carottages a permis de dégager un certain nombre de faciès sédimentologiques communs à l'ensemble du plateau nord-aquitain.

- Graviers et galets noirs constitués d'éléments siliceux et calcaires très arrondis, emballés dans une matrice de sable plus ou moins argileux avec de gros bioclastes très usés, perforés et encroûtés. Ce faciès révèle une grande hétérogénéité granulométrique, avec une médiane qui oscille entre 2 et $4 \mathrm{~mm}$ et une plurimodalité dont le mode principal se situe au-delà de $5 \mathrm{~mm}$.

- Sables grossiers (de 600 à $2000 \mu \mathrm{m}$ ) mal classés et composés d'éléments siliceux et de fragments bioclastiques enduits d'une patine de rubéfaction de couleur rousse. C'est un faciès bimodal dont le mode principal se situe autour de $300 \mu \mathrm{m}$.

- Sables moyens dont la couleur se situe entre le roux et le beige, suivant l'importance de la rubé- 
faction, présentant une grande homogénéité granulométrique. Ils sont toujours unimodaux (250 à 400 $\mu \mathrm{m})$ et bien classés.

- Sables fins à très fins qui, selon leur localisation, sont bimodaux (100 à $125 \mu \mathrm{m}$ et 315 à $400 \mu \mathrm{m})$ et riches en micas sur le proche plateau interne; ou, plus homogènes, unimodaux (160 à $200 \mu \mathrm{m})$ et bien classés, sur le plateau externe.

- Vases plus ou moins silteuses parfois associées aux faciès grossiers.

Il est possible de définir une séquence élémentaire constituée de trois termes [52]. Ainsi les sédiments sablo-graveleux forment le terme A de base sur une épaisseur moyenne d'environ 20 à $50 \mathrm{~cm}$; les sables moyens, terme B, constituent la part la plus importante en épaisseur des séquences élémentaires contenant le plus grand nombre et la plus grande diversité de structures sédimentaires. Le terme $\mathrm{C}$ est constitué, d'une part sur le plateau interne et médian, de sables moyens mais de granulométrie plus fine et mieux classée par rapport au terme B et, d'autre part sur le plateau externe, de sables fins gris pouvant contenir des passées vaso-silteuses. Nous verrons que le terme $\mathrm{C}$ se distingue surtout par la présence de figures sédimentaires de remaniement telles que des structures d'oscillation et des bioturbations.

Un certain nombre de caractères concernant la distribution verticale des dépôts peuvent être dégagés de l'étude de l'ensemble du plateau nord-aquitain, à savoir :

- la couverture superficielle est constituée d'un nombre restreint de séquences élémentaires superposées (en moyenne 2 ou 3 ) dont l'épaisseur diminue vers le sommet ;

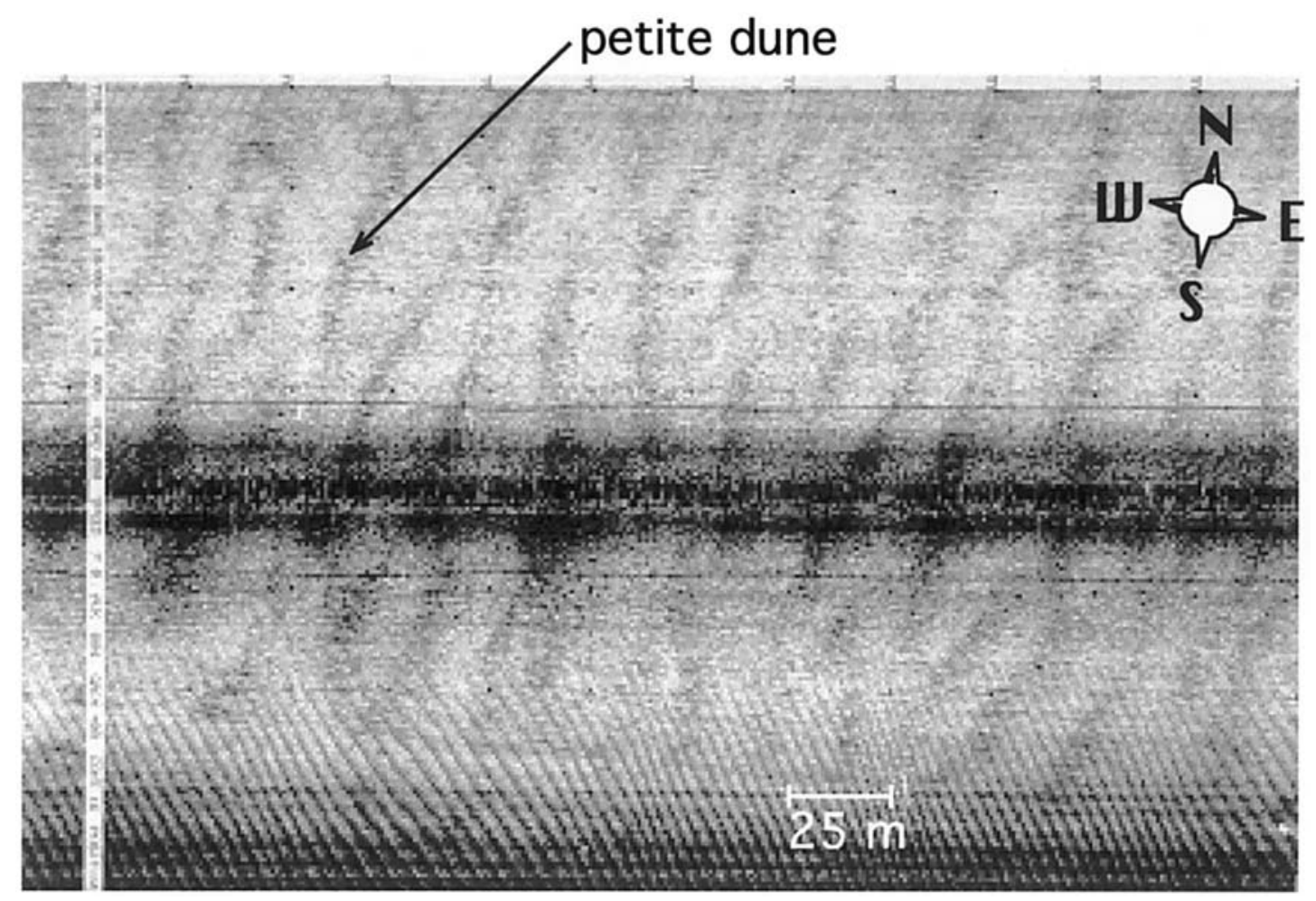

Figure 6. Photographie de sonogramme montrant des petites dunes sur le plateau interne nord-aquitain par environ $25 \mathrm{~m}$ de fond. Ces petites dunes $(\mathrm{H}=1,5 \mathrm{~m} ; \mathrm{L}=25 \mathrm{~m})$, de forme dissymétrique et arquée, dont le flanc raide, caractérisé par une granulométrie plus grossière (aspect plus foncé), est dirigé vers l'est, se déplaceraient vers le littoral.

Figure 6. Sonogram showing small dunes over the northern Aquitaine inner shelf at $25 \mathrm{~m}$ deep. These small dunes $(\mathrm{H}=1.5 \mathrm{~m} ; \mathrm{L}=25 \mathrm{~m})$ are dissymetric and curved with an abrupt flank of coarser sediment (dark shading) moving shoreward toward the east. 

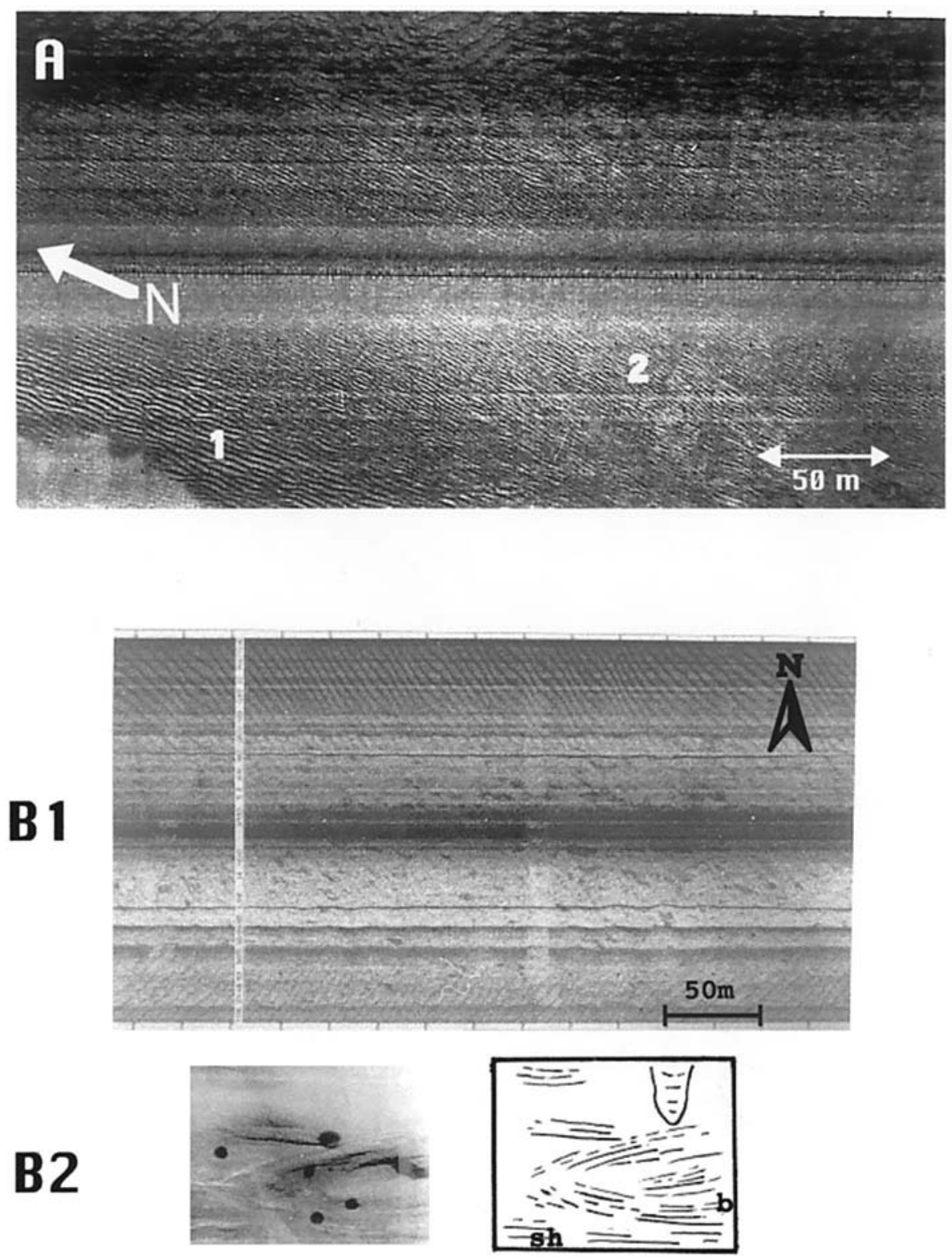

Figure 7. Formes sédimentaires liées aux mouvements oscillatoires de la houle (voir situation sur la figure 1). A : mégarides sur un substrat grossier, les crêtes rectilignes et bifurquées sont orientées nord-sud. On distingue deux générations de rides de forme symétrique : (1) de 2 à $3 \mathrm{~m}$ de longueur d'onde et probablement de plusieurs décimètres d'amplitude, (2) de 1 à $2 \mathrm{~m}$ de longueur d'onde et d'amplitude plus faible ; B1 : mégarides bombées hummocky megaripples sur fond de sables fins semblables à celles décrites par Reineck et Singh [39] sur le plateau est-américain ; B2 : radiographie et interprétation d'un box core prélevé sur ces fonds de sables fins et montrant des structures de type hummocky cross-stratification (hcs) parmi des structures sub-horizontales (sh).

Figure 7. Sedimentary bedforms linked with oscillatory flow from swell (see location on figure 1) A: Megaripples over a coarse substrate with their crest straight and bifurcated oriented north-south. Two generations of symetrical ripples can be distinguished: (1) wavelength $=$ 2 to $3 \mathrm{~m}$ and height $=$ several decimeters; (2) wavelength $=1$ to $2 \mathrm{~m}$ and height $=$ several centimeters; $\mathrm{B} 1$ : hummocky megaripples over fine sand similar to those described by Reineck and Sing [39] on the eastern American continental shelf; B2: radiography of a box core sampled in the fine sand deposit showing structures like hummocky cross-stratification (hcs) among sub-horizontal structures (sh). 


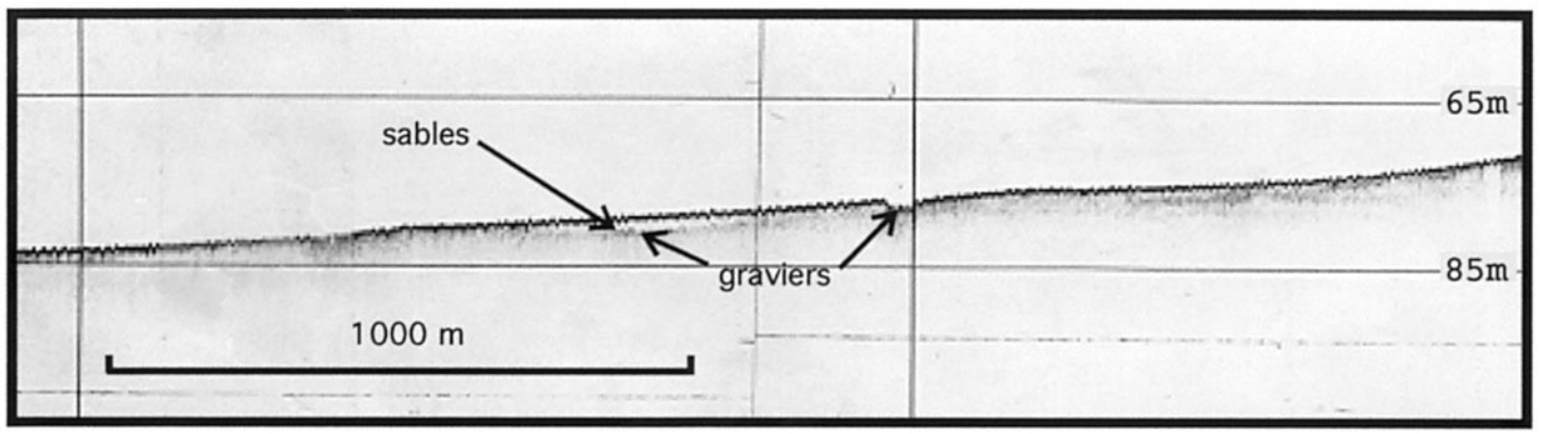

W

S.E.S.

Couverture superficielle = dépôts de remaniement du système de haut niveau

Soubassement $=$ dépôts du système transgressif

S.E.S. Surface d'érosion supérieure = surface d'inondation maximum

Figure 8. Portion de profil de sondeur de sédiment effectué sur le plateau nord-aquitain (voir situation sur la figure 1) montrant la géométrie de la couverture sédimentaire superficielle et replaçant cette dernière dans le cadre du modèle de stratigraphie génétique de Galloway [22].

Figure 8. Part of the echosounding profile on the North Aquitainian Shelf (see location on figure 1) showing the geometry of superficial sedimentary covering according to the model of genetic stratigraphy of Galloway [29].

- chaque séquence comprend également, à la base, une discontinuité sédimentaire érosionnelle, ce qui peut impliquer l'érosion partielle ou totale d'un ou de plusieurs termes sous-jacents ;

- la taille des structures sédimentaires (amplitude, épaisseur des sets) diminue de la base vers le sommet de chaque séquence;

- chaque séquence présente un grano-classement positif fining up.

Cependant, ces caractères généraux peuvent présenter, dans le détail, certaines variations caractéristiques de chaque zone du plateau continental. Afin de définir ces particularités, nous ne décrirons que quelques carottes parmi les plus représentatives.

Sur le proche plateau interne la carotte 8307 (figure $9 a$ ), située par $16 \mathrm{~m}$ de profondeur, comporte essentiellement une séquence élémentaire complète de 1,8 m d'épaisseur. Une telle épaisseur peut s'expliquer par le fait que la carotte a été prélevée dans la zone du prisme littoral où le stock sableux disponible et l'énergie hydrodynamique sont plus élevés que sur le reste du plateau.

Dans cette séquence unique, le terme A débute, à la base, par un contact érosif net, surmonté par 20 à $50 \mathrm{~cm}$ de sables moyens à grossiers $(300$ à $600 \mu \mathrm{m})$, très bioclastiques. Souvent ce niveau de base ne contient pas de structures apparentes. Cependant, il arrive que l'on observe des sets à lamines obliques d'une amplitude d'environ $10 \mathrm{~cm}$ et de fort pendage. Les bioclastes sont constitués, en grande partie, de valves de lamellibranches (Tellina tenuis et Solen marginatus) dont les faces concaves sont dirigées vers le haut. À cette faune sont associés des restes de gastéropodes (Clathrus clacthrus, Nassarius reticulatus) et des fragments de test d'échinodermes (Echinocyanus pusillus) caractéristiques de l'étage infra-littoral.

Le passage au terme B est progressif avec la mise en place de sables moyens (200 à $350 \mu \mathrm{m})$ légèrement 
bioclastiques. Les structures présentent, la plupart du temps, une succession de lits plans horizontaux, parfois légèrement obliques, de 2 à $3 \mathrm{~cm}$ d'épaisseur constitués par une alternance de sables moyens et de sables fins silteux. On constate que l'importance des lits de sable fin augmente progressivement vers le sommet au détriment de celle de sable moyen.

Le passage au terme $\mathrm{C}$ est graduel, conduisant à des sables fins silteux (100 à $200 \mu \mathrm{m})$ souvent micacés. Le classement granulométrique s'améliore progressivement vers le sommet de la séquence. Les structures sédimentaires sont essentiellement représentées par des lamines parallèles, sub-horizontales de 1 à $2 \mathrm{~mm}$ d'épaisseur. Dans les derniers décimètres, on observe fréquemment des stratifications obliques de faible amplitude qui sont à rattacher à des «rides de houle », en grande partie oblitérées par une intense bioturbation. Ainsi, cette partie terminale de la séquence correspond à un environnement relativement calme périodiquement mis en oscillation et vanné par la houle et dans lequel peut s'installer une activité biologique endobionte.

Sur le plateau interne proprement dit, la carotte VK8411, prélevée plus au large par $24 \mathrm{~m}$ de fond, est constituée par la superposition de trois séquences élémentaires (figure $9 b$ ). La séquence de base ne peut être appréhendée dans toute son épaisseur, car nous n'avons pu atteindre le niveau grossier du terme A, mais il apparaît en tout point identique à ce qui a été observé sur le proche plateau. En revanche, le terme B diffère au niveau des structures sédimentaires, constituées à la base par une stratification oblique, parfois bidirectionnelle, de 10 à $20 \mathrm{~cm}$ d'épaisseur et de pendage apparent $10^{\circ}$ à $15^{\circ}$. Les foresets sont de type " planaire tangentiel » parfois « sigmoïdal».

Encore plus au large, sur le plateau médian, la formation superficielle comprend, en général, une succession de deux à trois séquences élémentaires dont la séquence inférieure, comme précédemment, est la plus épaisse et de granulométrie plus grossière. Ces séquences sont constituées des trois unités présentant un grano-classement positif fining up.

Par rapport au plateau interne, on ne rencontre pas de différences très marquées. Celles-ci touchent principalement le terme A dans lequel la granulométrie est plus grossière (médiane entre 300 et $2000 \mu \mathrm{m}$ ). Les bioclastes sont également plus grands et plus épais, présentant des indices de reprises plus intenses et plus fréquentes (rubéfaction et usure). La faune paraît plus diversifiée mais elle caractérise toujours un étage infra-littoral (Venus, Cardium, Dosina, Echinocyanus pusillus, Spatangus sp., Cellaria).

Sur le plateau externe (carottes VK8314 et VK8320 de la figure 10), à partir de $80 \mathrm{~m}$ de fond, se développe une sédimentation devenant progressivement plus fine (sablo-silteuse). Les prélèvements présentent deux parties bien distinctes. Une série inférieure (I), dont on ne peut apprécier l'épaisseur, est organisée en « séquences mineures» de 10 à $30 \mathrm{~cm}$ d'épaisseur. Ces niveaux débutent par un contact érosif surmonté par des sables fins bioclastiques devenant vers le sommet progressivement plus silteux, puis vaseux. Les structures sédimentaires sont constituées de fines laminations horizontales ou très légèrement obliques. Ces petites séquences indiquent une brusque augmentation de la dynamique induisant des apports périodiques plus grossiers dans un environnement dominé par la sédimentation fine. Dans la partie supérieure (II), dont l'épaisseur varie de 0,5 à $2 \mathrm{~m}$, on distingue une succession de séquences élémentaires comparables à celles décrites pour le reste de la plate-forme. La différence réside dans le fait que la granulométrie des termes $\mathrm{B}$ et $\mathrm{C}$ est plus fine dans son ensemble (sables très fins, silts et vases).

\subsection{Datations}

La figure 11 représente, de façon schématique, la répartition des principaux carottages effectués le long d'une radiale ("Hourtin» sur la figure 1a) et leurs datations radiométriques (tableau II). Si l'on ne tient pas compte de quelques datations aberrantes, notamment pour la carotte VK 8416 (8130 ans BP : trop peu de matériel, de plus très remanié), on constate que :

- de la côte à la rupture de pente des $80 \mathrm{~m}$, de la carotte VK 8308 à la carotte 8418 , les âges sont compris entre l'actuel et -4000 ans BP, en accord avec les datations de Lesueur et al. [30], pour la base de la vasière ouest-Gironde; la mise en place des sédiments superficiels est donc relativement récente (Holocène supérieur). Arbouille [4] a montré que, durant cette période où le niveau marin demeurait constant et proche du niveau actuel, les apports sédimentaires étaient faibles ; 

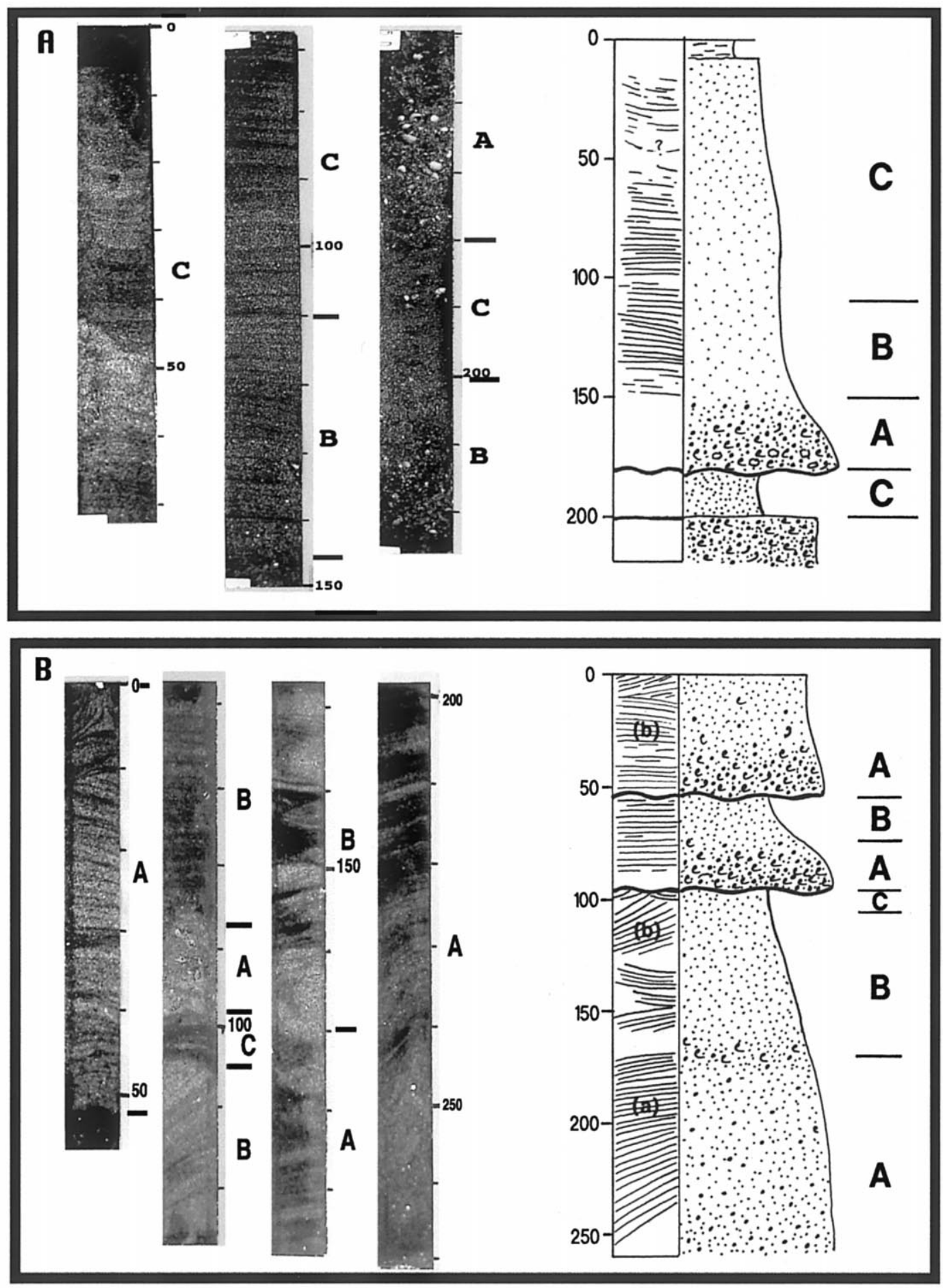

Figure 9. Les séquences élémentaires du plateau interne nord-aquitain : photographies et logs lithologiques (voir situation sur la figure 1 et légendes sur la figure 10). A : carotte VK8307 prélevée sur le proche plateau interne; B : carotte VK8411 prélevée sur le plateau interne proprement dit, (a) et (b) = structures «en éventail» décrites dans le texte.

Figure 9. Elementary sequences from the Inner North Aquitaine Continental shelf: photographs and lithological logs (see figures 1 and 10 for location and caption). A: core VK8307 sampled on the shoreface; B: core VK8411 sampled on the inner shelf, (a) and (b) fan-shaped structures (see text). 
- sur le plateau externe, de la carotte VK 8326 à la carotte VK 8318, les âges sont compris entre 5000 et 10000 ans BP dans la partie supérieure, et entre 20000 et 23000 ans BP dans le sédiment grossier résiduel de la base. Le matériel utilisé pour ces datations est constitué de valves de lamellibranches. La présence de structures sédimentaires dynamiques et l'aspect plus ou moins usé et brisé de certaines coquilles est un indice évident de remaniement, vraisemblablement très ancien, vue la profondeur actuelle supérieure à $90 \mathrm{~m}$. Les âges plus anciens correspondent à des dépôts du début de la transgression postglaciaire, voire des prismes de bas niveau dont certains peuvent être antérieurs au dernier glaciaire (figure 12).

La figure 12 regroupe toutes les datations au ${ }^{14} \mathrm{C}$ effectuées sur des valves de lamellibranches prélevées sur la plate-forme du golfe de Gascogne (tableau II), ainsi que deux courbes récentes de variation globale du niveau des océans depuis 25000 ans :

- celle proposée par Stanley [43] qui couvre la remontée des eaux au cours de l'Holocène ;

- celle de Linsley [31] qui donne également des informations sur la période de régression marine, avant 18000 ans.

Les données du plateau aquitain s'écartent évidemment de ces courbes, car les lamellibranches peuvent vivre sous plusieurs dizaines de mètres d'eau. Cependant, les données se répartissent de façon systématique au-dessous des courbes, confirmant d'une part, que le niveau marin s'est abaissé d'environ $110 \mathrm{~m}$ par rapport à l'Actuel [29] et montrant d'autre part, sur le plateau externe, la présence des sédiments très anciens, probablement antérieurs au dernier maximum glaciaire.

\section{DISCUSSION}

\subsection{Conditions hydrodynamiques générales actuelles}

Sur le plateau continental nord-aquitain, les courants de marée semi-diurnes, à caractère giratoire, sont faibles. Castaing [11] indique, par exemple, des vitesses de courant maximum dépassant rarement $25 \mathrm{~cm} \cdot \mathrm{s}^{-1}$ à $1 \mathrm{~m} \mathrm{du}$ fond. Ces courants ne permettent pas la remise en mouvement des sédiments déposés, mais seulement le transport par charriage des sédiments fins mis en oscillation par la houle.

Par rapport à la circulation générale atmosphérique, le golfe de Gascogne est situé dans la zone du vaste et intense courant d'ouest des moyennes latitudes. Il est donc directement exposé aux fortes houles engendrées dans l'Atlantique nord. De plus, c'est une zone où la
VK 8314

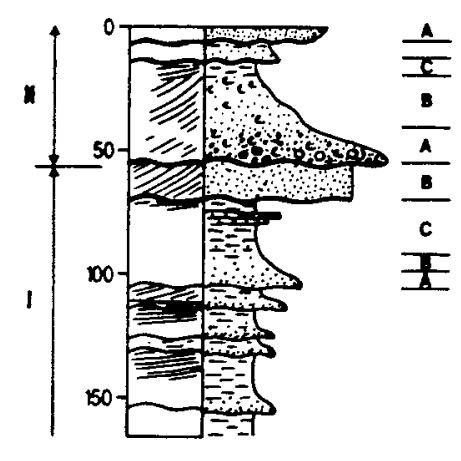

VK 8320

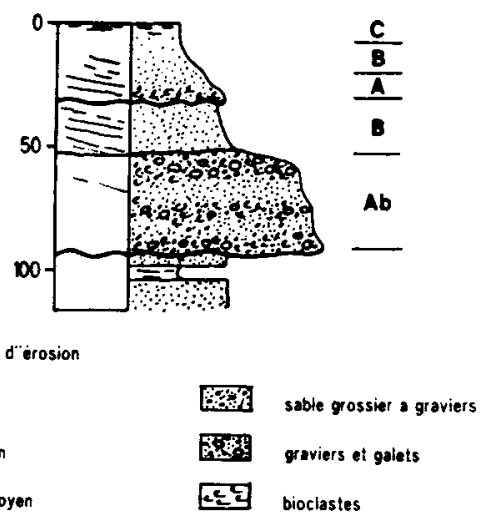

Figure 10. Séquences élémentaires du plateau externe nord-aquitain, exemple des carottes VK8314 et VK8320. Voir situation sur la figure 1; $\mathrm{Ab}=$ unité sédimentaire basale $($ cotes en $\mathrm{cm})$.

Figure 10. Elementary sequences of the external continental shelf, cores VK8314 and VK8320 as examples. See location on figure 1; $\mathrm{Ab}=$ basal sedimentary unit (in $\mathrm{cm}$ ). 


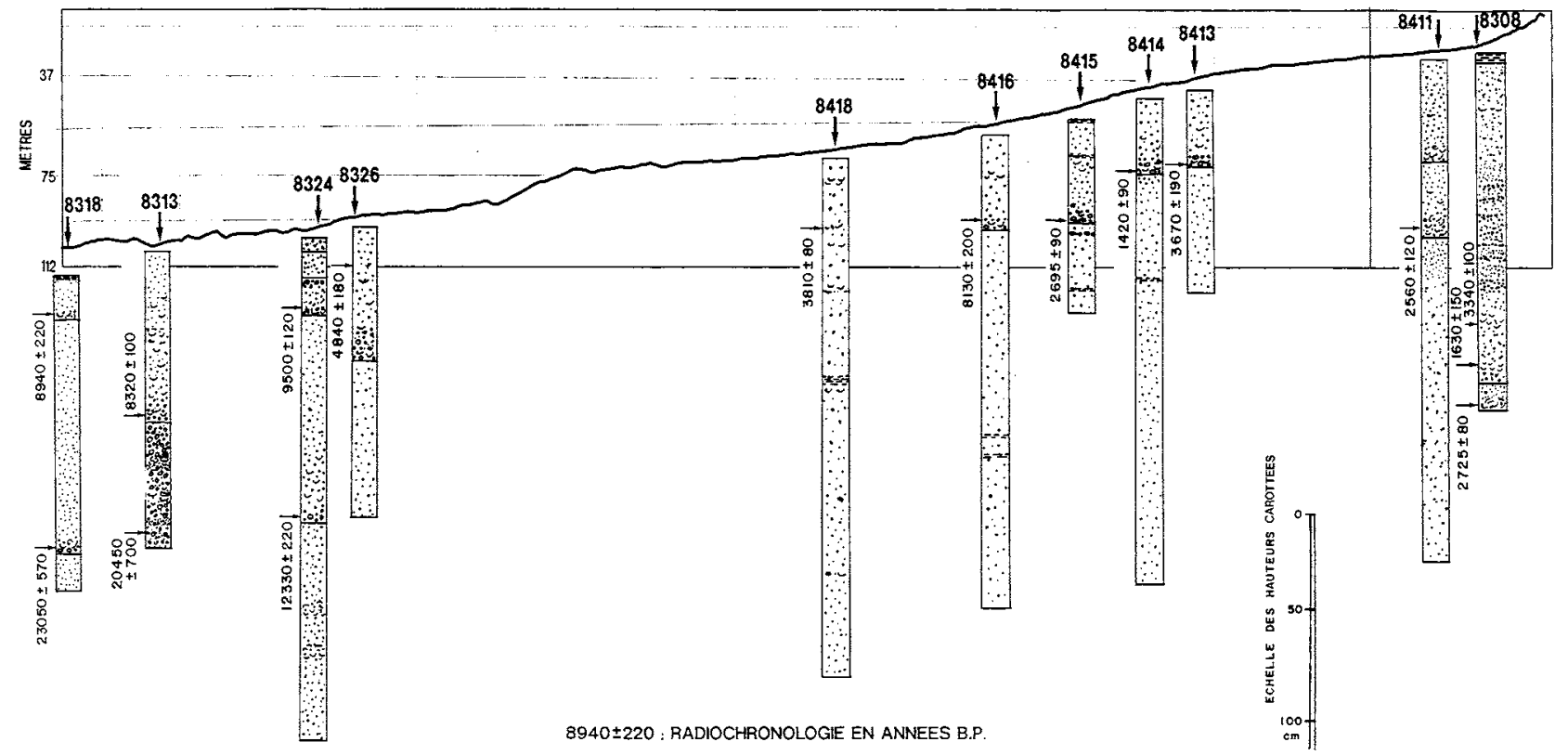

Figure 11. Répartition des principales carottes avec leurs datations radiochronologiques ${ }^{14} \mathrm{C}$ le long de la « radiale Hourtin» (cf. figure 1).

Figure 11. Distribution of the cores and ${ }^{14} \mathrm{C}$ radiological dating on the 'Hourtin transect' (see location on figure 1).

cyclogenèse est fréquente et rapide [51]. D'après Cavanié [12], les périodes des houles sont comprises entre $11 \mathrm{~s}$ et $15 \mathrm{~s}$. L'amplitude de $2 \mathrm{~m}$ est atteinte l'équivalent de trois mois par an; celle de $9 \mathrm{~m}$, l'équivalent d'un jour par an lors des tempêtes. La houle décennale a une amplitude de $15 \mathrm{~m}$ et la houle centennale, une amplitude qui se situerait entre 18 et $22 \mathrm{~m}$.

\subsection{Mise en mouvement des sédiments sous l'action de la houle}

D'après Castaing [11] et Barthe et Castaing [6], qui ont utilisé les données climatologiques et les abaques théoriques de mise en mouvement des sédiments, sous certaines conditions, tous les dépôts peuvent être mis en mouvement jusqu'au bord du talus sous l'action de la houle. Sur le plateau externe (profondeur supérieure à $100 \mathrm{~m}$ ) ces conditions sont rarement atteintes (houles de 15 à $20 \mathrm{~m}$ d'amplitude pour des périodes de $15 \mathrm{~s}$ ). En revanche, sur le plateau médian et interne (pour des profondeurs inférieures à $100 \mathrm{~m}$ ), les mises en mouvement peuvent se produire dès que l'amplitude dépasse $6 \mathrm{~m}$, ce qui est fréquent en hiver. Les traçages radioactifs [33], effectués sur la côte aquitaine, prouvent que jusqu'à $20 \mathrm{~m}$ de profondeur, les mouvements sédimentaires sont intenses. Au-delà, les volumes sédimentaires et la durée de mise en oscillation sont considérablement réduits.

\subsection{Intensité du remaniement des dépôts}

Dans les carottes, l'épaisseur des séquences élémentaires diminue de la côte vers le large [52], en liaison avec la diminution progressive mais rapide, pour des fonds inférieurs à $20 \mathrm{~m}$, du pouvoir de remaniement des processus hydrodynamiques. Depuis 4000 ans BP environ, le niveau de l'océan a peu varié dans le golfe de Gascogne [34]. Les apports sédimentaires par les fleuves sont très faibles et constitués exclusivement de 
Tableau II. Tableau des résultats de datations isotopiques ${ }^{14} \mathrm{C}$ effectuées sur des valves de lamellibranches prélevées sur le plateau continental du golfe de Gascogne (LGD : laboratoire de géologie dynamique, université Paris-VI ; LY : Centre de datation et d'analyses isotopiques, université Lyon-I ; Gif : LSCE, Gif-sur-Yvette; UQ : Geotop, univ. Montréal, Québec).

Table II. Results of ${ }^{14} \mathrm{C}$ dating obtained from bivalvia shells collected in the shelf sediment of the Bay of Biscay (LGD: laboratoire de géologie dynamique, université Paris-VI; LY: Centre de datation et d'analyses isotopiques, université Lyon-I; Gif: LSCE Gif-sur-Yvette; UQ: Geotop, univ. Montréal, Québec).

\begin{tabular}{|c|c|c|c|c|c|}
\hline $\mathrm{N}^{\circ} \mathrm{Ech}$. & Niveau $(\mathrm{cm})$ & Origine & Ref. & Ag (ans B.P.) & Prof $(\mathrm{m})$ \\
\hline VK 8308 & $150-155$ & FASEC & LGD & $3340 \pm 100$ & 25 \\
\hline VK 8308 & $166-170$ & FASEC & LY 3509 & $1630 \pm 150$ & 25 \\
\hline VK 8308 & $190-195$ & FASEC & LGD & $2725 \pm 80$ & 25 \\
\hline VK 8313 & $80-90$ & FASEC & LY 68 & $8320 \pm 180$ & 103 \\
\hline VK 8313 & $155-160$ & FASEC & LGD & $20450 \pm 700$ & 103 \\
\hline VK 8317 & $70-72$ & FASEC & LY 3673 & $9500 \pm 180$ & 103 \\
\hline VK 8318 & $17-20$ & FASEC & LY 3670 & $8940 \pm 220$ & 105 \\
\hline VK 8318 & $140-148$ & FASEC & LY 3674 & $23050 \pm 570$ & 105 \\
\hline VK 8323 & $150-155$ & FASEC & LGD & $13640 \pm 100$ & 102 \\
\hline VK 8324 & $31-33$ & FASEC & LGD & $9500 \pm 120$ & 92 \\
\hline VK 8324 & $150-160$ & FASEC & LY 3671 & $12330 \pm 220$ & 92 \\
\hline VK 8326 & $0-30$ & FASEC & LY 3667 & $4840 \pm 180$ & 92 \\
\hline VK 8411 & $90-95$ & FASEC & LY 3508 & $2560 \pm 120$ & 24 \\
\hline VK 8413 & $32-35$ & FASEC & LY 4047 & $3670 \pm 190$ & 35 \\
\hline VK 8414 & $38-40$ & FASEC & LGD & $1420 \pm 90$ & 42 \\
\hline VK 8415 & $41-50$ & FASEC & LGD & $2695 \pm 90$ & 48 \\
\hline VK 8416 & $49-51$ & FASEC & LGD & $8230 \pm 200$ & 56 \\
\hline VK 8417 & $38-42$ & FASEC & LY 3666 & $10290 \pm 310$ & 57 \\
\hline VK 8417 & 96-101 & FASEC & LY 3672 & $10200 \pm 390$ & 57 \\
\hline VK 8418 & $20-29$ & FASEC & LGD & $3810 \pm 80$ & 66 \\
\hline VK 8501 & $39-41$ & FASEC & LY 4005 & $10550 \pm 400$ & 120 \\
\hline VK 8501 & $88-90$ & FASEC & LY 4004 & $11280 \pm 280$ & 120 \\
\hline VK 8910 & $110-115$ & Lasalie & Gif-9081 & $3380 \pm 60$ & 31 \\
\hline VK 8909 & $115-125$ & Lasalie & Gif-9082 & $2210 \pm 40$ & 30 \\
\hline VK 8910 & $95-100$ & Lasalie & Gif-9083 & $5680 \pm 60$ & 31 \\
\hline VK 8910 & $115-120$ & Lasalie & Gif-9084 & $3440 \pm 60$ & 31 \\
\hline KC 8716 & 290 & Lesueur et al. (1996) [30] & LY 4444 & $1940 \pm 150$ & 65 \\
\hline KC 8708 & 244 & Lesueur et al. (1996) & LY 4445 & $3090 \pm 110$ & 45 \\
\hline KC 8705 & 205 & Lesueur et al. (1996) & Gif 7801 & $3920 \pm 70$ & 33 \\
\hline KC 8712B & 200 & Lesueur et al. (1996) & Gif 7799 & $4360 \pm 70$ & 50 \\
\hline $\mathrm{KC} \mathrm{87P3}$ & 305 & Lesueur et al. (1996) & LY 4441 & $4360 \pm 110$ & 50 \\
\hline $\mathrm{KC} 87 \mathrm{P} 3$ & 305 & Lesueur et al. (1996) & LY 4442 & $4950 \pm 750$ & 50 \\
\hline КC 9084 & 129 & Lesueur et al. (1996) & UQ 1975 & $1130 \pm 122$ & 43 \\
\hline \multirow[t]{8}{*}{ KC 9084} & $130-140$ & Lesueur et al. (1996) & UQ 1973 & $5780 \pm 122$ & 43 \\
\hline & & Glemarec & In Barusseau (1973) [7] & 15000 & 150 \\
\hline & & Lapierre & In Barusseau (1973) & 26000 & 200 \\
\hline & & Lapierre & In Barusseau (1973) & 11000 & 118 \\
\hline & & Lapierre & In Barusseau (1973) & 7100 & 50 \\
\hline & & Lapierre & In Barusseau (1973) & 5000 & 32 \\
\hline & & Radiocarb. & In Barusseau (1973) & $6350 \pm 160$ & 40 \\
\hline & & Radiocarb. & In Barusseau (1973) & $10200 \pm 230$ & 110 \\
\hline RBO 3T & & Barusseau (1973) [7] & Gif & $4650 \pm 115$ & 78 \\
\hline RBO 12T & & Barusseau (1973) & Gif & $2250 \pm 100$ & 89 \\
\hline RBO 8T & & Barusseau (1973) & Gif & $10700 \pm 190$ & 85 \\
\hline RBO 57T & & Barusseau (1973) & Gif & $1400 \pm 90$ & 65 \\
\hline $\mathrm{RBO} 45 \mathrm{~T}$ & & Barusseau (1973) & Gif & $4900 \pm 115$ & 40 \\
\hline THE 139 & & Barusseau (1973) & Lyon & $19960 \pm 400$ & 41 \\
\hline THE 139 & & Barusseau (1973) & Lyon & $17400 \pm 450$ & 41 \\
\hline THE 22 & & Barusseau (1973) & Lyon & $6080 \pm 200$ & 32 \\
\hline THE 22 & & Barusseau (1973) & Lyon & $6280 \pm 400$ & 32 \\
\hline THE 128 & & Barusseau (1973) & Lyon & $8240 \pm 220$ & 49 \\
\hline IRC 1-6 & & Barusseau (1973) & Paris & $10800 \pm 450$ & 163 \\
\hline IRC 13A-9 & & Barusseau (1973) & Paris & $10630 \pm 500$ & 160 \\
\hline
\end{tabular}




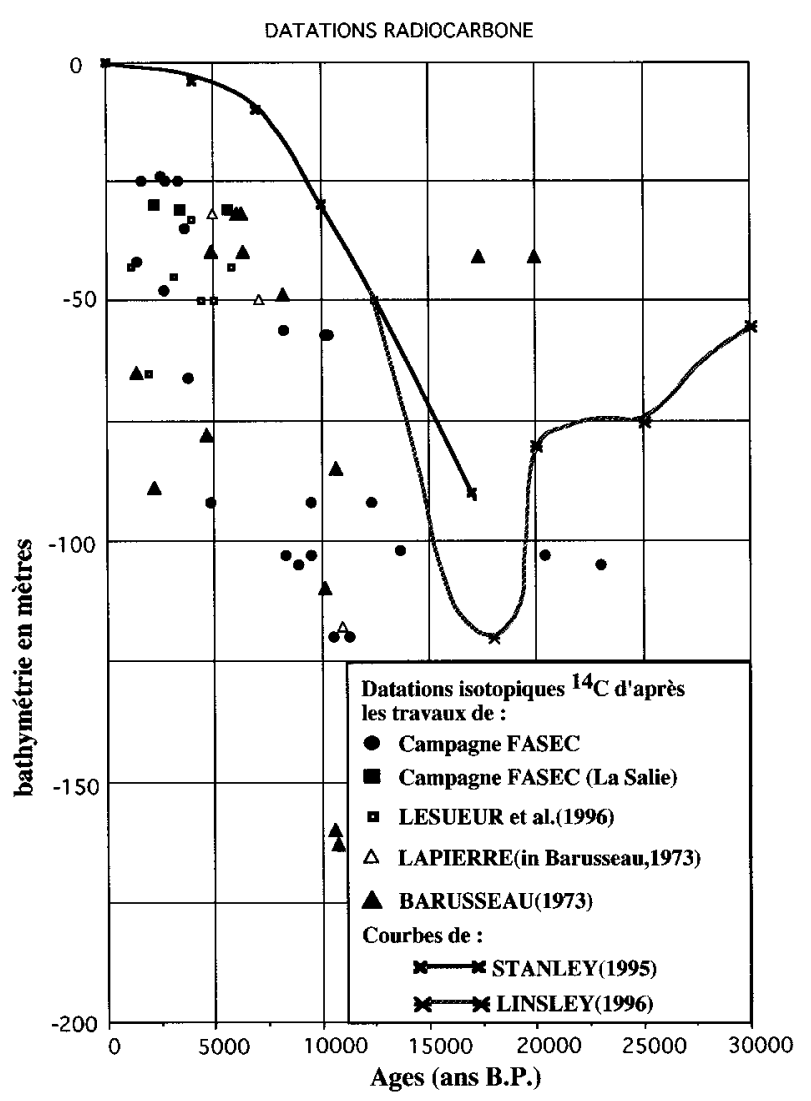

Figure 12. Âges absolus de valves de lamellibranches prélevées sur la plate-forme du golfe de Gascogne d'après différents travaux et position de ces datations par rapport aux courbes de variation du niveau des océans depuis 25000 ans BP (courbes de Stanley [43] et de Linsey [31]).

Figure 12. Absolute ages from bivalvia shells recovered on the Bay of Biscay Shelf from various authors and position of these datings with reference to the curve of sea level changes since $25000 \mathrm{y} \mathrm{BP}$ (curve from Stanley [43] and Linsey [31]).

matériaux fins (silts et argiles) qui sédimentent dans la vasière "ouest-Gironde», ou sont directement transportés au-delà du talus continental. Les matériaux sableux constituant les fonds du plateau interne, comme ceux du reste de la plate-forme, sont en place depuis beaucoup plus de 4000 ans et soumis à des remaniements, essentiellement par la houle. Dans la zone littorale, une partie du sable provient de l'érosion de la côte [4]. Quant aux datations, ces remaniements se traduisent de deux façons: a) par une grande hétérogénéité dans l'âge absolu des sédiments carottés sur le plateau interne à des pro- fondeurs peu différentes (carottes 8413 et 8414 par exemple sur la figure 11); b) par des intercalations de sédiments récents au sein de sédiments plus anciens (carotte 8303 par exemple sur la figure 11). Il serait aberrant d'évaluer des taux de sédimentation entre 4000 ans BP et aujourd'hui sur le plateau interne. En revanche l'examen de la position dans la carotte, des datations inférieurs ou égales à 4000 ans BP permet d'évaluer l'épaisseur de remaniement des dépôts au cours de cette période. À titre d'exemple, sur la carotte 8308 longue de $2 \mathrm{~m}$ (figure 11), les datations donnent $2725 \pm 80$ ans à la base et $3340 \pm 100$ ans à $40 \mathrm{~cm}$ au-dessus, montrant qu'à la profondeur de $25 \mathrm{~m}$ le remaniement par la houle s'effectue au moins sur $2 \mathrm{~m}$ d'épaisseur. À l'inverse, à partir de $90 \mathrm{~m}$, les dates sont plus homogènes, et toutes plus anciennes que 4000 ans BP, même à proximité de la surface. Ceci indique d'une part, l'absence depuis longtemps d'apport sableux sur cette partie du plateau et d'autre part, que la houle et les courants sur le fond ne remanient pas ou peu les sédiments sableux. La prise en compte de l'ensemble des datations dans toutes les carottes et de leur cote par rapport à la surface (tableau II) permet d'établir la courbe d'épaisseur du remaniement en fonction de la profondeur d'eau pour les 4000 dernières années. Le principe d'établissement de la courbe est simple. Il consiste, pour une profondeur donnée, à repérer sur les carottes, la cote maximale par rapport au fond où se trouvent des sédiments dont l'âge est inférieur à 4000 ans BP. Cette cote représente la limite vraisemblable du remaniement par les agents dynamiques depuis que le niveau de l'océan est à peu près stationnaire dans le golfe de Gascogne. En effet, en l'absence de toute sédimentation, le dépôt en profondeur de ces sédiments sableux ne peut résulter que du remaniement. Pour les faibles valeurs de remaniement se pose la question d'un enfouissement lié au mode de vie des lamellibranches sur lesquels sont effectuées les datations. Cette hypothèse est à écarter car les datations ont été pratiquées sur des débris coquilliers ou des valves isolées, et non en position de vie.

La courbe montre (figure 13) que l'intensité du remaniement diminue rapidement avec la profondeur, surtout sur le plateau interne. Ainsi, à $25 \mathrm{~m}$ de profondeur, l'épaisseur de remaniement est supérieure à $200 \mathrm{~cm}$; elle n'est plus que de $120 \mathrm{~cm}$ à $30 \mathrm{~m}$. L'épaisseur remaniée est encore significative à 
$50 \mathrm{~m}$ (de l'ordre de $40 \mathrm{~cm}$ ); à partir de $80 \mathrm{~m}$, elle devient très faible (de l'ordre de $10 \mathrm{~cm}$ ). Au-dessous de $90 \mathrm{~m}$, dès la surface, l'âge des sédiments est supérieur à 4000 ans $(4840$ ans \pm 180 à $92 \mathrm{~m}$ pour la carotte 8326).

Ces données obtenues à partir des datations ${ }^{14} \mathrm{C}$ sont intéressantes dans la mesure où elles corroborent les observations actuelles et les estimations à partir des abaques [11].

\subsection{Action des courants de tempête sur l'organisation interne des dépôts superficiels}

Le tableau de la figure 14 synthétise la succession des séquences élémentaires qui constituent les dépôts superficiels du plateau nord-aquitain avec leurs caractéristiques structurales et texturales ainsi que les conditions hydrodynamiques de leur mise en place $[11,15]$.

On constate en général une diminution globale de la granulométrie de la côte vers le large. Le passage des sables moyens beiges aux sables fins gris se situe approximativement à la limite des plateaux médian et externe (entre 80 et $100 \mathrm{~m}$ de profondeur). Cependant, nous avons fréquemment observé que les unités grossières (graviers et galets) rencontrées à la base des carottages sur le plateau externe, se caractérisent par des médianes bien plus élevées $(\geq 3000 \mu \mathrm{m})$ que sur le reste du plateau. Il est probable que ceci soit lié à la proximité, dans cette zone, des dépôts grossiers de remplissage de bas niveau marin.

Toutes les séquences élémentaires (figure 14) débutent par un contact érosif avec la séquence précédente. Ce contact exprime une phase érosive initiale résultant d'un courant à haute énergie capable de remettre en mouvement une certaine quantité de sédiment.

On constate, au sein des séquences élémentaires, une évolution verticale de la nature et de la dimension des structures sédimentaires. L'épaisseur des faisceaux ainsi que celle des lamines diminue progressivement vers le sommet des séquences. Les structures obliques dont le pendage apparent se situe, en moyenne, entre $10^{\circ}$ et $20^{\circ}$, correspondent à des sets de mégarides édifiées par un courant tractif unidirectionnel. Ces structures obliques prennent, vers le sommet, une disposition tangentielle, voire sigmoïdale, puis passent à des structures sub-horizontales et/ou à de petites structures obliques d'oscillation liées à la houle et souvent associées à une intense bioturbation. Reading [38], dans son chapitre traitant des plateformes siliciclastiques, met cette évolution en rapport avec une diminution de l'énergie cinétique sur le fond, diminution qui provoque un changement dans les processus de transit sédimentaire. Quant à la disposition des structures obliques en «éventail», nous avons vu précédemment qu'elle pouvait être liée au déplacement du corps sédimentaire et/ou à une rotation progressive du sens du courant tractif. Des photographies $\mathrm{du}$ fond (non publiées) permettent de confirmer que les placages sableux étaient parcourus par des rides symétriques d'oscillation dont la longueur d'onde se situe entre 30 et $50 \mathrm{~cm}$. Selon Clifton et Dingler [15], il est possible de calculer, en fonction de la longueur d'onde des rides, de la granulométrie du sédiment et de la bathymétrie, l'amplitude et la période des houles qui ont provoqué ces mégarides. Ainsi sur le plateau médian nord-aquitain, les mégarides sur les sables ont été vraisemblablement engendrées par des houles dont la période se situerait entre 8 et $9 \mathrm{~s}$ et l'amplitude entre 4 et $5 \mathrm{~m}$. De telles houles se rencontrent au moins l'équivalent de $30 \mathrm{j}$ par an sur le plateau nord-aquitain. Enfin, des mesures de courantométrie effectuées à $5 \mathrm{~m}$ du fond au large d'Arcachon, ont donné des vitesses maximales de $40 \mathrm{~cm} \cdot \mathrm{s}^{-1}$ pouvant mettre en mouvement des sables moyens [11]. Les sables grossiers et les graviers sont souvent associés, en surface, à des mégarides de longueur d'onde métrique (figures 4 et 7). Ces mégarides, dont le rapport «amplitude longueur d'onde » vaut à peu près 0,15 , se sont mises en place sous l'action de courants oscillatoires. Pour un sédiment grossier dont la moyenne granulométrique est d'environ $1 \mathrm{~mm}$, la houle doit avoir 15 à $16 \mathrm{~s}$ de période et $15 \mathrm{~m}$ d'amplitude, valeurs élevées mais non exceptionnelles à l'échelle du siècle sur le plateau aquitain.

Chaque séquence élémentaire représente l'enregistrement d'un seul événement hydrodynamique comprenant au moins trois phases:

1) Une phase érosive initiale, correspondant au paroxysme de la tempête, au cours de laquelle une partie plus ou moins importante de la séquence sous-jacente est érodée. Cet événement est engendré par un processus hydrodynamique intense, capable de trans- 
porter par charriage et saltation des particules dont le diamètre dépasse 500 à $1000 \mu \mathrm{m}$, ce qui nécessite des courants unidirectionnels sur le fond de l'ordre de 60 à $100 \mathrm{~cm} \cdot \mathrm{s}^{-1}$. Selon Castaing [11], ce sédiment peut être mis en mouvement sur le plateau interne et médian (jusqu'à $75 \mathrm{~m}$ de profondeur) par des tempêtes annuelles ou décennales $(0,1$ à $9 \mathrm{j}$ par an).

2) Une phase de transport et de dépôt correspondant à l'amortissement progressif de la tempête et aux courants de fond qui lui sont associés. Au cours de cette phase se déposent d'abord des sédiments grossiers, sablo-graveleux, comprenant une forte proportion de bioclastes, puis des sables moyens consti-

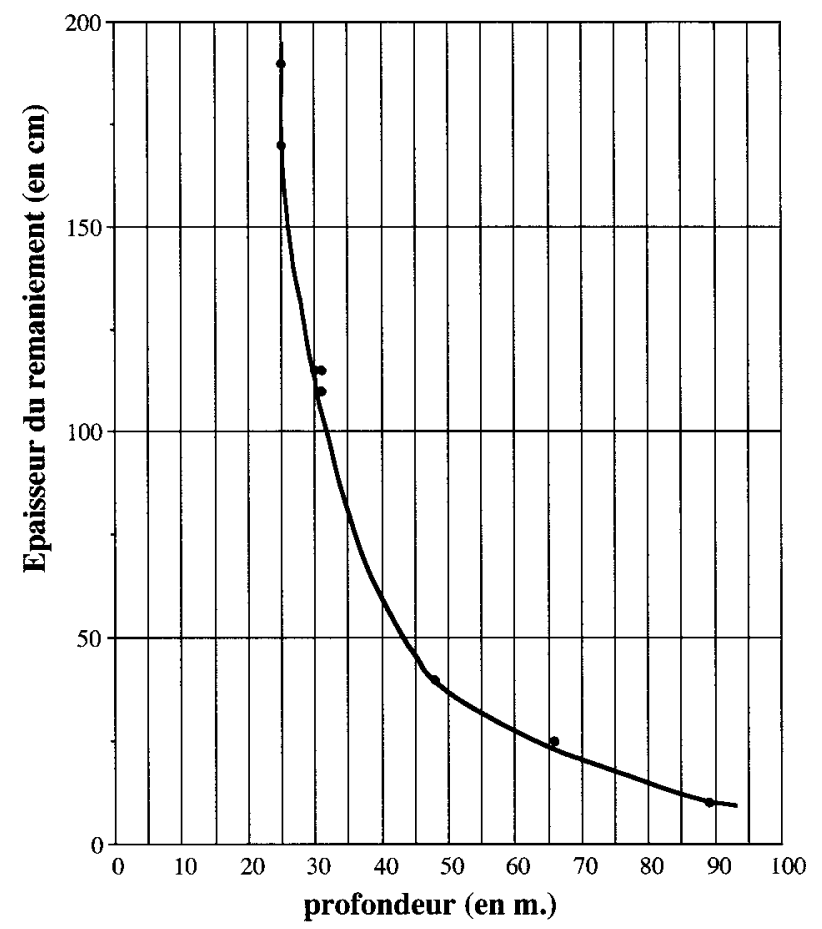

Figure 13. Évaluation de l'épaisseur du remaniement des sédiments sableux sur le plateau aquitain en fonction de la profondeur depuis 4000 ans BP (pour une profondeur donnée, le point matérialise la cote maximale sous le fond de l'océan où l'on trouve, dans les carottes, des sédiments d'âge inférieur à 4000 ans $\mathrm{BP})$.

Figure 13. Evaluation of the depth of reworking of sandy sediments on the Aquitaine Shelf related to depth since 4000 y BP (for a given water depth, the point shown represents the maximum depth beneath the seabed where sediments of an age of less than 4000 years were found). tuant des foresets tangentiels ou angulaires dont l'amplitude diminue progressivement vers le sommet où l'on passe à des structures sub-horizontales.

3) Une ou plusieurs phases de remobilisation/décantation résultant, en grande partie, des mouvements oscillatoires induits par la houle, soit à la fin d'une forte tempête lorsque subsiste une houle résiduelle suffisamment ample; soit, plus tard, lors du passage d'une autre tempête moins violente ou d'un grand train de houle d'ouest. Cette ultime phase produit le vannage de la partie superficielle de la séquence, conduisant à un sédiment relativement fin (sables moyens à fins pouvant contenir des intercalations vaseuses produite lors des phases de décantation). Le sédiment est ordonné en structures la plupart du temps planaires horizontales, passant dans la partie superficielle de la séquence à des structures symétriques d'oscillation fortement bioturbées. Il convient de souligner que la formation basale (figure 14), constituée de graviers, galets et gros bioclastes, ne peut être mise en mouvement que par des courants de vitesses supérieures à $200 \mathrm{~cm} \cdot \mathrm{s}^{-1}$, inconcevables sur le plateau aquitain. Ce type de sédiment, peu remanié, comme en témoignent les encroûtements sur les bioclastes, résulte surtout de phénomènes de vannage conduisant à un dépôt résiduel lag deposit. Il est logique de penser que cette formation résiduelle se prolonge sous les placages sableux et correspond au réflecteur de base signalé plus haut (figure 8 ) pouvant affleurer localement à la faveur des dépressions et des sillons érosifs.

\subsection{Action des processus hydrodynamiques sur la répartition des dépôts superficiels}

Sur le plateau aquitain, la reconnaissance en imagerie sonar fait apparaitre des formes sédimentaires en relation directe avec l'hydrodynamisme de tempête. Elles sont représentées par des sillons ou des couloirs de graviers et de sables grossiers allongés parallèlement à la côte et qui, pour la plupart des auteurs [10, 26, 32], seraient des formes érosives longitudinales. Ces couloirs sont bordés par des placages sableux rubanés, constitués d'un empilement de séquences de dépôt de tempête.

Nous proposons de schématiser les processus responsables de cette disposition selon le bloc-diagramme de 


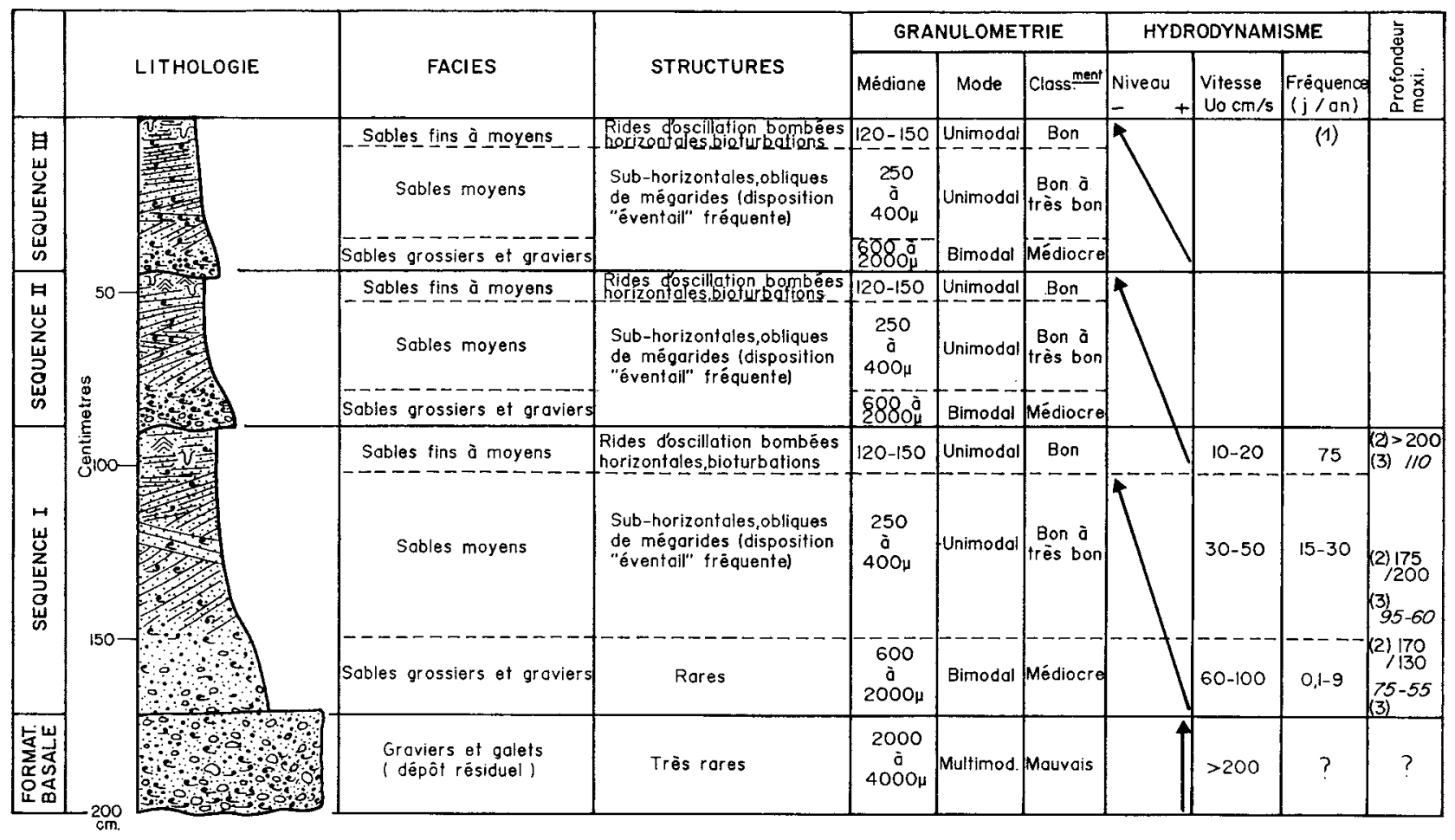

Figure 14. Schéma de la superposition des séquences élémentaires synthétiques qui constituent les dépôts superficiels du plateau nord-aquitain avec leurs caractéristiques structurales et texturales ainsi que les conditions hydrodynamiques qui peuvent leur être associées d'après les travaux de Castaing [11]. $\mathrm{U}_{0}=$ vitesse orbitale au fond $\mathrm{cm} \cdot \mathrm{s}^{-1} ;(1)=$ temps de mise en oscillation par une houle de $12 \mathrm{~s}$ de période à $60 \mathrm{~m}$ de profondeur ; $(2)=$ profondeur maximale de mise en oscillation par des houles de tempêtes exceptionnelles $(\mathrm{H}=22 \mathrm{~m}$, $\mathrm{T}=15 \mathrm{~s}) ;(3)=$ profondeur maximale de mise en oscillation par des houles de tempêtes « annuelles » $(\mathrm{H}=9 \mathrm{~m}, \mathrm{~T}=12 \mathrm{~s})$.

Figure 14. Schematic representation of the superposition of synthetic elementary sequences forming superficial deposits on the Aquitainian Shelf with their structural and textural characteristics and the possible hydrodynamic conditions that could be associated [11]. $\mathrm{U}_{0}$ $\mathrm{cm} \cdot \mathrm{s}^{-1}=$ bottom orbital velocity $\mathrm{cm} \cdot \mathrm{s}^{-1}$; (1) oscillation time for a wave with a period of $12 \mathrm{~s}$ at $60 \mathrm{~m}$ depth; (2) maximum depth of oscillation for decadal storm waves $(\mathrm{H}=22 \mathrm{~m}, \mathrm{~T}=15 \mathrm{~s})$; (3) maximum depth of oscillation for annual storm waves $(\mathrm{H}=9 \mathrm{~m}, \mathrm{~T}=12 \mathrm{~s})$.

la figure 15. Sur le plateau nord-aquitain, les transits sédimentaires sont très réduits. Ils résultent, en grande partie, des puissants courants combinés sur le fond, induits par les tempêtes. C'est probablement dans ces phases dynamiques paroxysmales que se produit l'essentiel de la remobilisation du sédiment. Durant la phase d'amortissement qui suit, la compétence des courants diminue progressivement, la composante liée aux courants orbitaux de la houle ou à la force de Coriolis prend de l'importance et provoque la déviation du courant. Le sédiment se dépose latéralement en séquences granoclassées de tempête. Le reste du temps, la propagation des fortes houles d'ouest et de nord-ouest (à partir de $4 \mathrm{~m}$ d'amplitude) n'affecte que la partie supérieure $(10$ à $20 \mathrm{~cm})$ de la couverture sédimentaire en engendrant des mégarides d'oscillation.

La figure 16 montre, de façon schématique, la répartition des principales formes sédimentaires. Sur les replats morphologiques du plateau médian, la couverture sableuse superficielle s'étale de façon quasi continue, ne laissant apparaître le substrat grossier qu'à la faveur d'occasionnelles fenêtres d'érosion. Sur la partie du plateau interne comprenant le prisme littoral, où cette couverture est la plus épaisse, le substrat grossier n'apparait que rarement. En surface, la présence de petites dunes est fréquente. En revanche, sur les zones de plus forte pente (abrupts morphologiques), la couverture sableuse est plus irrégulière et présente les formes sédimentaires décrites 


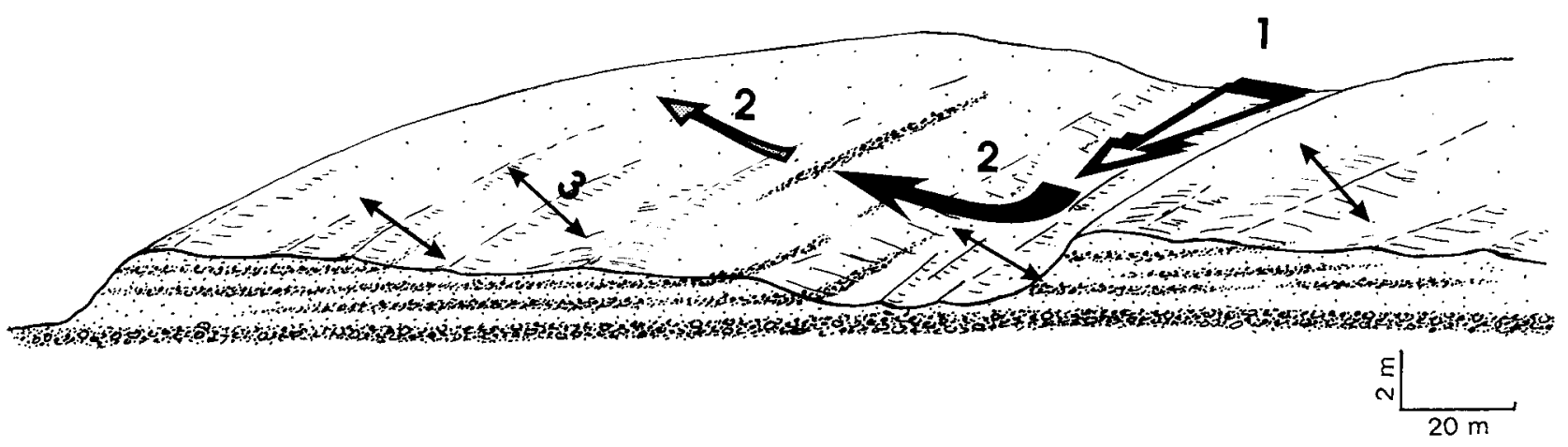

Figure 15. Schéma de la dynamique sédimentaire récente sur le plateau continental nord-aquitain. 1 : courant tractif sur le fond, à caractère érosif induit par les tempêtes; 2 : courant dévié dont la perte de compétence progressive entraîne un dépôt granoclassé au niveau des placages sableux latéraux ; 3 : mouvements oscillatoires induits, par la suite, par les fortes houles.

Figure 15. Interpretation of the recent sediment dynamics on the north Aquitaine Continental Shelf. 1: erosive current at bottom generated by storm; 2: deviated current with a progressive diminution of competence giving graded bedding deposits on the lateral sand patches; 3: oscillatory motions caused, secondarily, by strong waves.

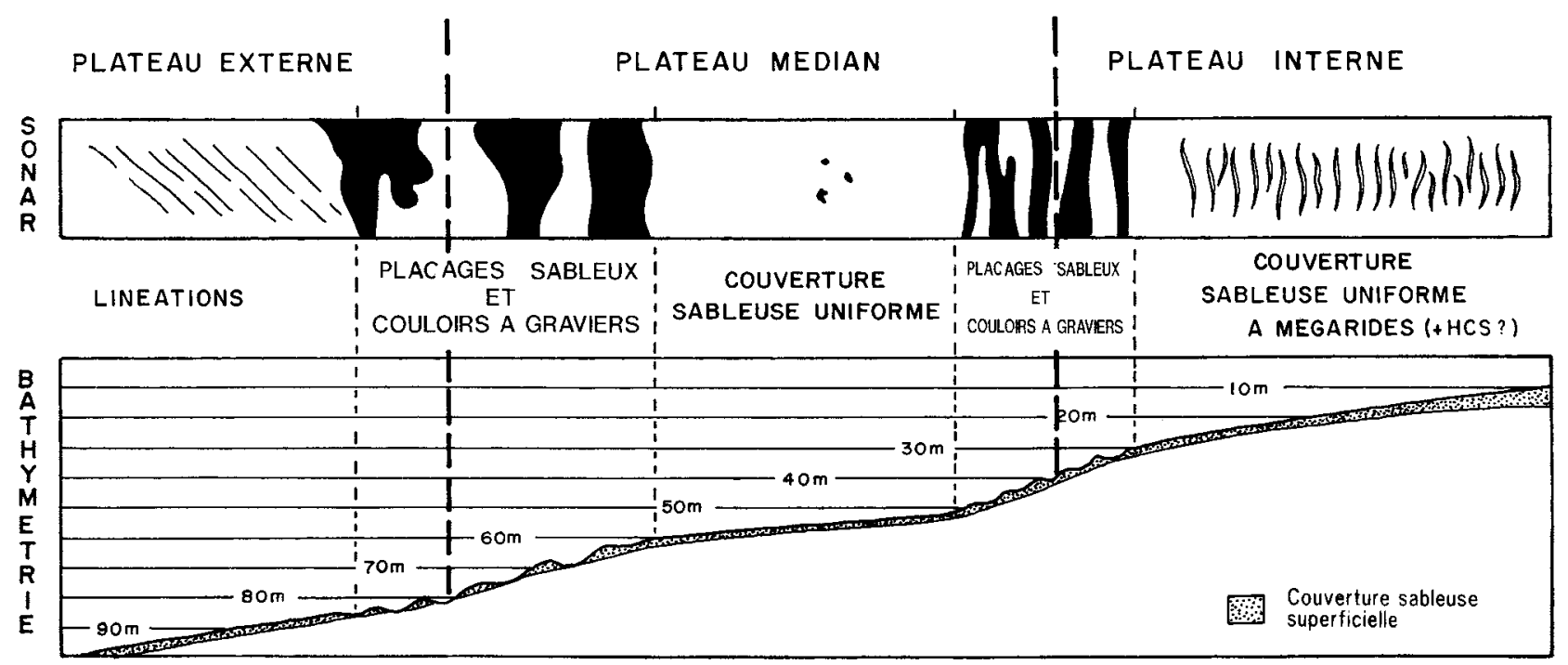

Figure 16. Synthèse de la répartition des formes sédimentaires sur le plateau nord-aquitain $(\mathrm{HCS}=$ hummocky cross-stratifications $=$ stratifications en mamelons).

Figure 16. Summary representation of the distribution of the bedforms on the North Aquitaine Continental Shelf (HCS = hummocky cross-stratification).

précedemment, couloirs de type érosionnel alternant avec des "rubans" de placage sableux. Les sables fins du plateau externe sont affectés par des linéations ou sillons érosifs, rectilignes et orientés WNW-ESE.

La plate-forme nord-aquitaine présente donc une couverture sédimentaire superficielle dominée par un régime d'accommodation au sens de Swift et Thorne [48]. La sédimentation récente est essentiellement autochtone, caractérisée par des remaniements répétés des stocks sédimentaires anciens conduisant à la mise en place de dépôts résiduels. 
Cet article constitue la contribution DGO-UMR/Epoc $\mathrm{n}^{\circ} 1305$

\section{RÉFÉRENCES}

[1] Allen, G.P., Castaing, P., 1977. Carte de répartition des sédiments superficiels sur le plateau continental du golfe de Gascogne. Bull. IGBA 21, 255-260.

[2] Allen, J.R.L., 1982. Sedimentary structures: their character and physical basis, Development in Sedimentology, vol. 30. Elsevier, p. 663.

[3] Aloïsi, J.C., Auffret, G.A., Auffret, J.P., Barusseau, J.P., Hommeril, P., Larsonneur, C., Monaco, A., 1977. Essai de modélisation de la sédimentation actuelle sur les plateaux continentaux français. Bull. Soc. Géol. France 19, 183-195.

[4] Arbouille D., 1987. La sédimentation de la plate-forme continentale nord-aquitaine au Quaternaire terminal: un exemple de système transgressif, thèse $3^{\mathrm{e}}$ cycle, univ. Bordeaux-I, 211 p.

[5] Auffret J.P., Berne S., Gresselin F., D’Ozouville J.L., Walker P., 1986. Anse de Vauville: géologie et dynamique sédimentaire, notice explicative de la carte Ifremer au 1/5000.

[6] Barthe, X., Castaing, P., 1989. Étude théorique de l'action des courants de marée et des houles sur les sédiments du plateau continental du golfe de Gascogne. Oceanol. Acta 12, 325-334.

[7] Barusseau J.P., 1973. Évolution du plateau continental rochelais (golfe de Gascogne) au cours du Pliocène terminal et de l'Holocène, les processus actuels de la sédimentation, thèse univ. Bordeaux-I.

[8] Berné, S., Allen, G., Auffret, J.P., Chamley, H., Durand, J., Weber, O., 1989. Essai de synthèse sur les dunes hydrauliques géantes tidales actuelles. Bull. Soc. Géol. France 6, 11451160.

[9] Boczar-Karakiewicz, B., Bona, J.L., 1986. Wave-dominated shelves: a model of sand-ridge formation by progressive, infragravity waves. In: Knight, R.J., McLean, J.R. (éds.), Shelf sands and sandstones, Can. Soc. Petrol. Geol. Mem, vol. 11, pp. 163-179.

[10] Cacchione, D.A., Drake, D.E., Grant, W.D., Tate, G.B., 1984. Rippled scour depressions on the inner continental shelf off central California. J. Sed. Petrol. 54, 1280-1291.

[11] Castaing P., 1981. Le transfert à l'océan des suspensions estuariennes, Cas de la Gironde, thèse d'état univ. Bordeaux-I, $530 \mathrm{p}$.

[12] Cavanié, A., 1975. Application à la zone au large du Finistère d'une méthode d'estimation statistique des courants de houle au voisinage du fond. La Houille Blanche 7/8, 497-499.

[13] Channon, R.D., Hamilton, D., 1976. Wave and tidal-current sorting of shelf sediments SW of England. Sedimentology 23, $17-32$.

[14] Cirac, P., Berné, S., Lericolais, G., Weber, O., 1997. Séquences de dépôt dans le Quaternaire terminal du plateau continental nord-aquitain (océan Atlantique, France). Bull. Soc. Géol. France 168, 717-725.
[15] Clifton, H.E., Dingler, J.R., 1984. Wave-formed structures and paleo-environmental reconstruction. Mar. Geol. 6, 165198.

[16] Collotte P., 1985. Le plateau central nord-aquitain, caractérisation morphosédimentaire et interprétation dynamique des dépôts holocènes, thèse $3^{\mathrm{e}}$ cycle, univ. Bordeaux-I, $278 \mathrm{p}$.

[17] Dalrymple, R.W., 1984. Morphology and internal structure of sand waves in the Bay of Fundy. Sedimentology 31, 365-382.

[18] Draper, J.L., 1967. Wave activity at the sea bed around NW Europe. Mar. Geol. 5, 133-140.

[19] Flemming, B.W., 1981. Factors controlling shelf sediment dispersal along the SE African continental margin. Mar. Geol. 42, 259-277.

[20] Flemming, B.W., 1988. Pseudo-tidal sedimentation in a nontidal shelf environment (SE African continental margin). In: de Boer, P.L., et al. (éds.), Tide-Influenced Sedimentary Environments and Facies. D. Reidel Publ. Co, pp. 167-180.

[21] Forbes, D.L., Boyd, R., 1987. Gravel ripples on the inner Scotian shelf. J. Sed. Petrol. 57, 46-54.

[22] Galloway, W.E., 1989. Genetic stratigraphic sequences in basin analysis I: architecture and genesis of flooding surface bounded depositional units. Am. Ass. Petrol. Geol. Bull. 73, $125-142$.

[23] Guillocheau F., 1990. Stratigraphie séquentielle des bassins de plate-forme: l'exemple dévonien armoricain, thèse doct. sci. nat., univ. Louis-Pasteur-Strasbourg, 257 p.

[24] Harms J.C., Southard J.B., Walker R.G., 1982. Structures and sequences in clastic rocks, SEPM Short course number 9, 2d ed., Tulsa, Oklahoma, 239 p.

[25] Hunter, R.E., Dingler, J.R., Anima, R.J., Richmond, B.M., 1988. Coarse-sediment bands on the inner shelf of Southern Monterey bay, California. Mar. Geol. 80, 81-98.

[26] Karl, H.A., 1980. Speculations on processes responsible for mesoscale current lineations on the continental shelf, Southern California. Mar. Geol. 34, 9-15.

[27] Kenyon, N.H., 1970. The origin of some transverse sand patches in the Celtic Sea. Geol. Mag. 107, 389-394.

[28] Knebel, H.J., Needell, S.W., O'Hara, C.J., 1982. Modern sedimentary environments on the Rhode Island inner shelf, off the Eastern United States. Mar. Geol. 49, 241-256.

[29] Lapierre F., 1969, Répartition des sédiments sur le plateau continental du golfe de Gascogne, intérêts des minéraux lourds, thèse, univ. Bordeaux-I.

[30] Lesueur, P., Tastet, J.P., Marambat, L., 1996. Shelf mud fields formation within historical times: examples from offshore the Gironde estuary, France. Cont. Shelf Res. 16, 1849-1870.

[31] Linsley, B.K., 1996. Oxygen-isotope record of sea level and climate variations in the Sulu sea over the past 150000 years. Nature 380, 234-237.

[32] McKinney, T.F., Stubblefield, W.L., Swift, D.J.P., 1974. Large-scale current lineations on the central New Jersey shelf: investigations by side-scan sonar. Mar. Geol. 17, 79-102.

[33] Migniot, C., 1977. Action des courants, de la houle et du vent sur les sédiments. La Houille Blanche 1, 9-47. 
[34] Morzadec-Kerfourn M.T., 1995. Coastline changes in the Armorican Massif (France) during the Holocene, in: Finkl C.W. (éd.), Holocene cycle: climate, sea level and sedimentation, J. Coast. Res., Sp. Issue, 197-203.

[35] Newton, R.S., Seibold, E., Werner, F., 1973. Facies distribution patterns on the Spanish Sahara continental shelf mapped with the side scan sonar. Meteor Forsch.-Ergebn. 15, 55-57.

[36] Nummedal D., 1991. Shallow marine storm sedimentation, The oceanographic perspective, in: Einsele et al. (éd.), Cycles and events in stratigraphy, Springer-Verlag, pp. 227-255.

[37] Penin F., 1980. Le prisme littoral aquitain : histoire holocène et évolution récente des environnements morphosédimentaires, thèse $3^{\mathrm{e}}$ cycle, univ. Bordeaux-I, $129 \mathrm{p}$.

[38] Reading, H.G., 1986. Sedimentary environments and facies, 2nd ed. Blackwell Sci. Publ, p. 615.

[39] Reineck, H.E., Singh, I.B., 1980. Depositional sedimentary environments. Springer-Verlag, p. 549.

[40] Roy, P.S., Thom, B.G., Wright, L.D., 1980. Holocene sequences on an embayed high energy coast: an evolutionary model. Sedim. Geol. 26, 1-19.

[41] Snedden, J.W., Nummedal, D., 1991. Origin and geometry of storm-deposited sand bed in modern sediments of the Texas continental shelf. In: Swift, D.J.P., Oertel, G.F., Tillman, R.W., Thorne, J.A. (éds.), Shelf sand and sandstone bodies: Geometry, facies and sequence stratigraphy. Blackwell, pp. 283-308.

[42] Snedden, J.W., Nummedal, D., Amos, A.F., 1988. Storm and fair-weather combined flow on the central Texas continental shelf. J. Sed. Petrol. 58, 580-595.

[43] Stanley, D.J., 1995. A global sea-level curve for the Late Quaternary: the impossible dream. Mar. Geol. 125, 1-6.

[44] Swift, D.J.P., Figueiredo, A.G., Freeland, G.L., Oertel, G.F., 1983. Hummocky cross-stratification and megaripples: a geological double standard. J. Sed. Petrol. 53 (4), 1295-1317.

[45] Swift, D.J.P., Freeland, G.L., 1978. Current lineations and sand waves on the inner shelf, Middle Atlantic Bight of North America. J. Sed. Petrol. 48 (4), 1257-1266.

[46] Swift, D.J.P., Freeland, G.L., Young, R.A., 1979. Time and space distribution of meggaripples and associated bedforms, Middle Atlantic Bight, North American Atlantic shelf. Sedimentology 26, 389-406.

[47] Swift, D.J.P., Han, G., Vincent, C.E., 1986. Fluid process and sea floor response on a modern storm-dominated shelf: middle Atlantic shelf of North America, Part 1: the storm current regime. In: Knight, R.J., McLean, J.R. (éds.), Shelf sands and sandstones, Can. Soc. Petrol. Geol., Mem, vol. 11, pp. 99119.

[48] Swift, D.J.P., Thorne, J.A., 1991. Sedimentation on continental margins, I: a general model for shelf sedimentation. In: Swift, D.J. P., Oertel, G.F., Tillman, R.W., Thorne, J.A. (éds.), Shelf sand and sandstone bodies: Geometry, facies and sequence stratigraphy. Blackwell, pp. 3-31.

[49] Turcq B., 1984. Faciès et formes sédimentaires du plateau continental nord-aquitain, réponse aux processus hydrodynamiques actuels, thèse $3^{\mathrm{e}}$ cycle, univ. Bordeaux-I.

[50] Turcq, B., Cirac, P., Berné, S., Weber, O., 1986. Caractéristiques des environnements sédimentaires de la plate-forme continentale nord-aquitaine en relation avec les processus hydrodynamiques actuels. Bull. IGBA 39, 149-164.

[51] Vassal J.P., 1980. Les houles exceptionnelles et leurs conséquences, estimation des conditions maximales d'état de la mer sur le golfe de Gascogne. Analyse d'une forte tempête récente : 30 nov. -5 déc. 1976, thèse, univ. Bordeaux-I, $119 \mathrm{p}$.

[52] Weber, O., Arbouille, D., Faugères, J.C., 1987. Séquences sédimentaires granoclassées de haute énergie sur une plateforme continentale à niveau marin constant : exemple de la plate-forme atlantique du Nord de l'Aquitaine (France). C.R. Acad. Sci., série II 304 (19), 1191-1194.

[53] Weber O., Legigan P., Turcq B., Berné S., Cirac P., Collotte P., 1983. Étude de la couverture meuble du plateau nordaquitain : rapport $\mathrm{CFP} / \mathrm{IGBA} \mathrm{n}^{\circ} \mathrm{DE} / 351$. 